\title{
Resolving the Complexity of Ubiquitin Networks
}

\author{
Katarzyna Kliza*t and Koraljka Husnjak* \\ Institute of Biochemistry II, Medical Faculty, Goethe University, Frankfurt, Germany
}

Ubiquitination regulates nearly all cellular processes by coordinated activity of ubiquitin writers (E1, E2, and E3 enzymes), erasers (deubiquitinating enzymes) and readers (proteins that recognize ubiquitinated proteins by their ubiquitin-binding domains).

\section{OPEN ACCESS}

Edited by:

Monique P. C. Mulder,

Leiden University Medical Center,

Netherlands

Reviewed by:

Jun Yin,

Georgia State University,

United States

Robert Layfield,

University of Nottingham,

United Kingdom

*Correspondence:

Katarzyna Kliza

K.Kliza@science.ru.nl

Koraljka Husnjak

K.Husnjak@

biochem2.uni-frankfurt.de

tPresent address:

Katarzyna Kliza,

Department of Molecular Biology,

Faculty of Science, Radboud Institute for Molecular Life Sciences, Oncode

Institute, Radboud University

Nijmegen, Nijmegen, Netherlands

Specialty section

This article was submitted to

Cellular Biochemistry,

a section of the journal

Frontiers in Molecular Biosciences

Received: 22 October 2019

Accepted: 04 February 2020

Published: 27 February 2020

Citation:

Kliza K and Husnjak K (2020) Resolving the Complexity of Ubiquitin Networks. Front. Mol. Biosci. 7:21. doi: 10.3389/fmolb.2020.00021
By differentially modifying cellular proteome and by recognizing these ubiquitin modifications, ubiquitination machinery tightly regulates execution of specific cellular events in space and time. Dynamic and complex ubiquitin architecture, ranging from monoubiquitination, multiple monoubiquitination, eight different modes of homotypic and numerous types of heterogeneous polyubiquitin linkages, enables highly dynamic and complex regulation of cellular processes. We discuss available tools and approaches to study ubiquitin networks, including methods for the identification and quantification of ubiquitin-modified substrates, as well as approaches to quantify the length, abundance, linkage type and architecture of different ubiquitin chains. Furthermore, we also summarize the available approaches for the discovery of novel ubiquitin readers and ubiquitin-binding domains, as well as approaches to monitor and visualize activity of ubiquitin conjugation and deconjugation machineries. We also discuss benefits, drawbacks and limitations of available techniques, as well as what is still needed for detailed spatiotemporal dissection of cellular ubiquitination networks.

Keywords: ubiquitin, ubiquitin receptor, affinity purification, mass spectrometry, E3 ligase, deubiquitinating enzyme

\section{INTRODUCTION}

Post-translational modifications (PTMs) greatly increase the complexity and functional diversity of the proteome, ensuring rapid and dynamic cellular responses to the environmental and intracellular factors (Walsh et al., 2005). Extensive research over the last few decades has revealed elaborate control of a variety of cellular processes by a small protein ubiquitin (Ub), including cellular proteostasis, DNA repair, trafficking and immunity (Varshavsky, 2006; Kulathu and Komander, 2012).

$\mathrm{Ub}$ is a small, highly compact globular protein, with the exception of its unrestrained and flexible C-terminal tail (Figure 1A). To achieve high cellular Ub concentrations, 4 different genes (UBB, UBC, RPS27, and UBA52) encode Ub in mammals. Genes UBB and UBC encode linear fusions of 3 and $9 \mathrm{Ub}$ molecules, respectively, whereas RPS27A and UBA52 encode Ub as in-frame fusion to a small and large ribosomal protein, respectively (Figure 1B) (Ozkaynak et al., 1984; Finley et al., 1989).

Protein modification by Ub (ubiquitination) occurs through the formation of the covalent bond between $\alpha$-carboxyl group of the terminal glycine (Gly) residue of Ub and, typically, $\varepsilon$-amino group 


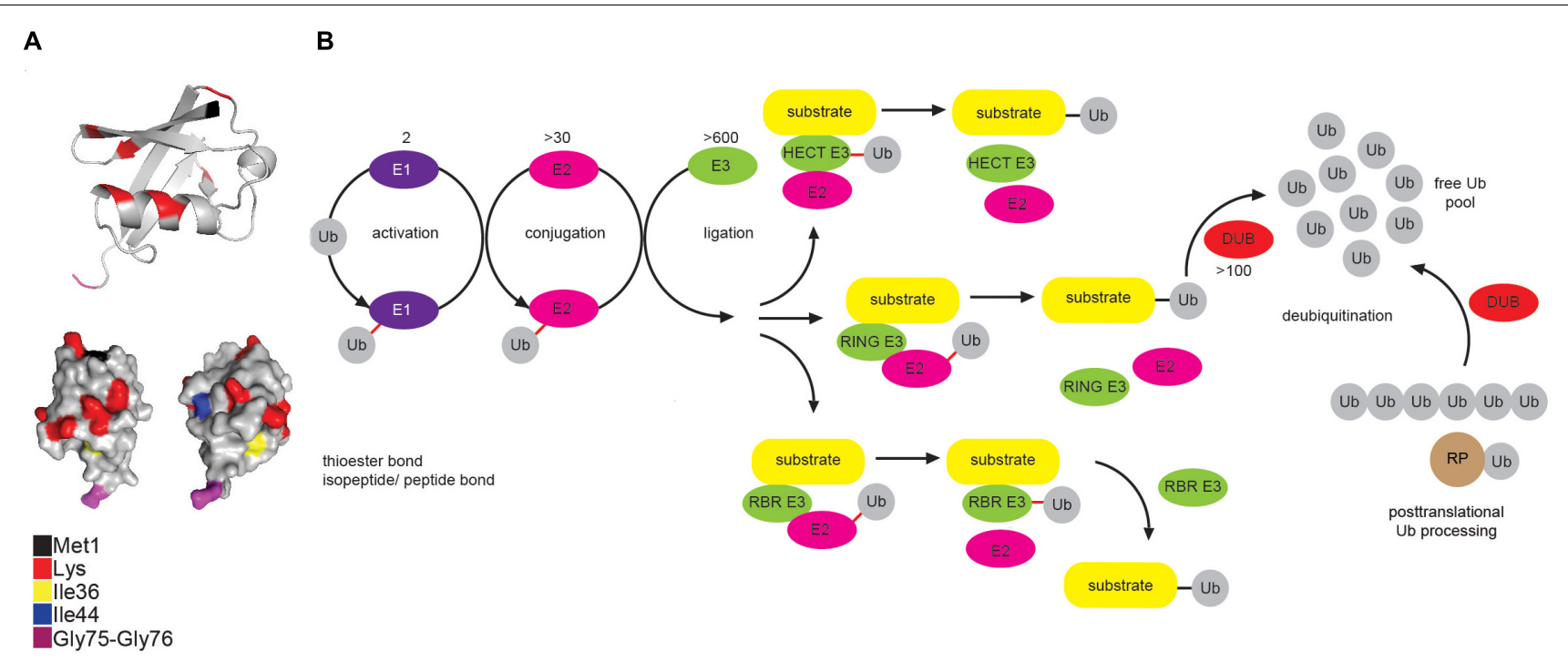

FIGURE 1 | Multicomponent enzymatic machineries assemble and disassemble ubiquitin modification. (A) Ub belongs to the $\beta$-grasp fold ( $\beta$-GF) family (Vijay-Kumar et al., 1987), in which $\beta$-GF is formed by five-stranded $\beta$-sheet, a short $3_{10}$ helix and a 3.5 -turn $\alpha$-helix. The C-terminal Ub tail is essential for Ub conjugation and hence, for all the Ub functions. Functionally relevant Ub residues are depicted in different colors. The figure was generated from the PDB entry 1 UBQ by PyMOL v1.7.6.0 software. (B) Coordinated activity of Ub-activating (E1), Ub-conjugating (E2), and Ub-ligating enzyme (E3) is required for Ub attachment to substrate protein. The action modes of the three main groups of E3 ligases (RING, HECT and RBR) are also depicted. In mammals, Ub is encoded by four different genes: UBA52 and RPS27A genes encode a single Ub molecule fused to the ribosomal subunits $L 40$ and S27a, respectively (depicted as RP-Ub), UBB and UBC genes encode 2 different polyUb precursor proteins (exemplified here as $U_{6}$ fusion). More than 100 cellular DUBs process newly translated Ub-containing polypeptides, remove Ub from modified substrates and disassemble unanchored Ub chains.

of an internal lysine (Lys) residue of the substrate. Interestingly, some mammalian and viral E3 ligases target thiol group of cysteine (Cys) residue (Cadwell and Coscoy, 2005; Williams et al., 2007), whereas a subset of substrates, such as ataxin3 and tau, is modified by the attachment of $\mathrm{Ub}$ to an $\alpha$-amino group of their $\mathrm{N}$-terminal residues, in a process known as N-terminal ubiquitination (Ciechanover and BenSaadon, 2004). Additionally, serine (Ser) and threonine (Thr) residues can also function as ubiquitination sites, forming hydroxyester bonds between $\mathrm{Ub}$ and target proteins (McDowell and Philpott, 2016) and thus expanding the biological importance of ubiquitination even further.

Ubiquitination is achieved by a coordinated and sequential enzymatic cascade (Figure 1B). Classically, $\mathrm{Ub}$ is activated in an ATP-dependent reaction by an Ub-activating (E1) enzyme and subsequently transferred to the active Cys residue of an Ub-conjugating (E2) enzyme, followed by $\mathrm{Ub}$ attachment to a substrate mediated by an Ub-ligating (E3) enzyme. Until now, two E1s, nearly $30 \mathrm{E} 2 \mathrm{~s}$ and over $600 \mathrm{E} 3 \mathrm{~s}$ have been identified in humans. Mechanistically, E3 ligases belong to either RING (really interesting new gene), HECT (homologous to E6-AP C terminus or RBR (RING-between-RING, hybrid RING-HECT) classes and can generate Ub linkages of different length and architecture (Metzger et al., 2012; Walden and Rittinger, 2018).

The activity of ubiquitination machinery can be reversed by more than 100 deubiquitinating enzymes (DUBs), which hydrolyze isopeptide or peptide bond resulting in $\mathrm{Ub}$ deconjugation from the ubiquitinated protein (Figure 1B) (Komander et al., 2009; Mevissen and Komander, 2017). DUBs affect cellular pool of free Ub by releasing newly synthesized $\mathrm{Ub}$ from Ub precursors, removing non-essential Ub molecules and recycling $\mathrm{Ub}$ from the former ubiquitination events (Reyes-Turcu et al., 2009; Grou et al., 2015).

\section{Different Forms of Ubiquitin Modifications Exist in Nature}

Cellular Ub modifications occur in various forms, which are usually referred to as "Ub code." Modification by a single $\mathrm{Ub}$ moiety (monoubiquitination) is the most abundant $\mathrm{Ub}$ modification that regulates DNA repair, transcription, signal transduction, viral budding, endocytosis and even proteasomal degradation (Chen and Mallampalli, 2009; Braten et al., 2016). After $\mathrm{Ub}$ is transferred to the $\varepsilon$-amino group of a target Lys, any of the eight amino groups of Ub (Met1, Lys6, Lys11, Lys27, Lys29, Lys33, Lys48, Lys63) can be attached to the $\mathrm{C}$ terminus of another $\mathrm{Ub}$ to form $\mathrm{Ub}$ chain of variable length, linkage type and configuration (homo- and heterotypic/branched $\mathrm{Ub}$ chains). Even though functional significance of several Ub modifications (such as Lys48- and Lys63-linked ubiquitination) is largely known, the biological significance of other $\mathrm{Ub}$ modifications is still far from being fully understood (Figure 2). Amongst the homotypic Ub chains, Lys48-linked Ub polymers were historically first identified and are predominant among homotypic polyUb chains (Peng et al., 2003; Swatek and Komander, 2016). These Ub linkages mark proteins for proteolytic degradation, which in turn regulates signal transduction, cell division, stress response, adaptive 


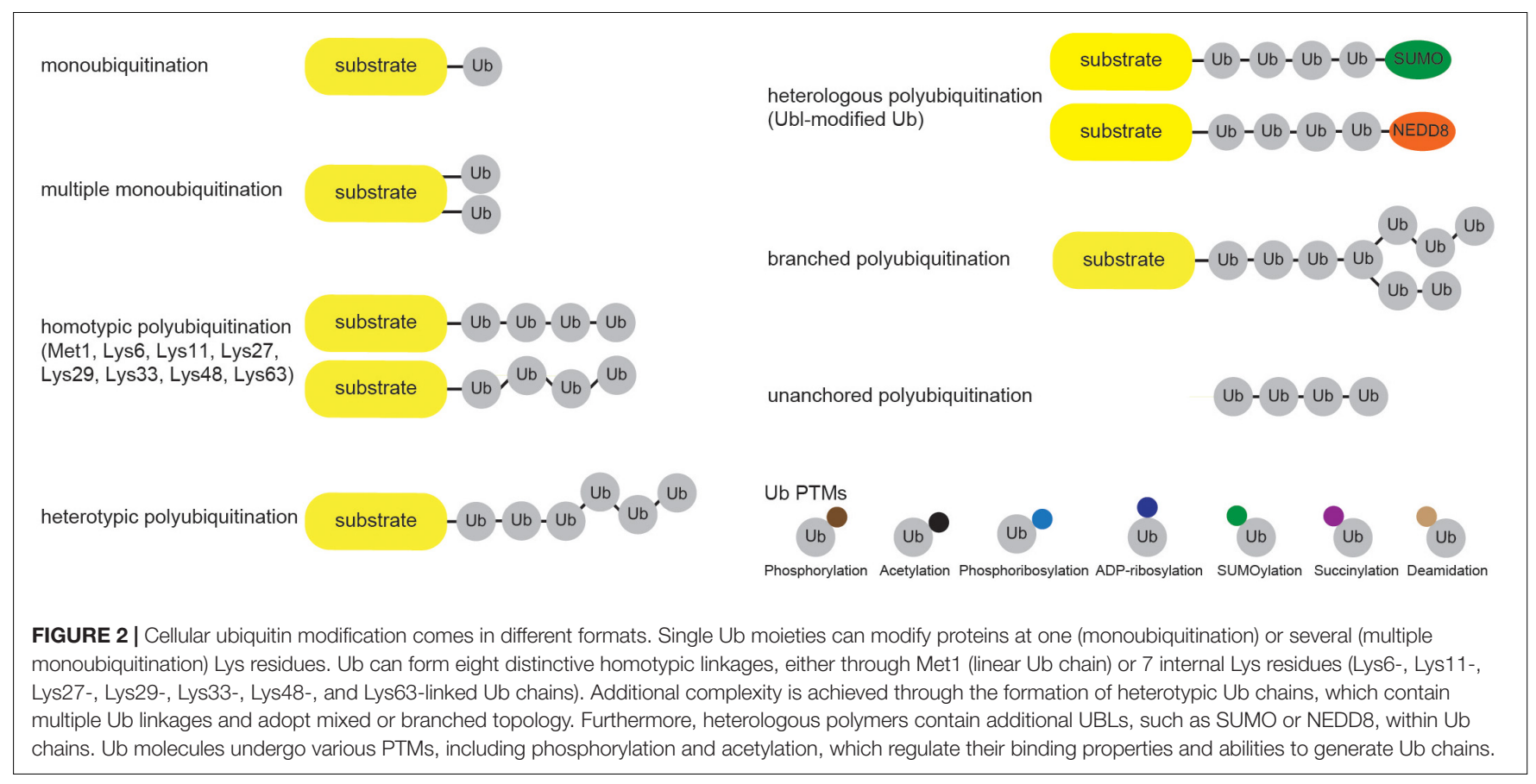

immune system and development (Hershko and Ciechanover, 1998; Wang and Maldonado, 2006; Park et al., 2007). The remaining homotypic polyUb chains are collectively called atypical (Liu et al., 2015; Swatek and Komander, 2016), and their physiological roles are nicely summarized elsewhere (Kulathu and Komander, 2012; Akutsu et al., 2016). Branched Lys11/Lys48 and hybrid Met1/Lys63 linkages were recently implicated in proteasomal degradation and $\mathrm{NF \kappa B}$ signaling, respectively (Emmerich et al., 2013; Meyer and Rape, 2014; Grice et al., 2015).

Besides by ubiquitination, Ub molecules can also be modified by acetylation, phosphorylation, ADP-ribosylation, phosphoribosylation, deamidation, SUMOylation and succinylation (Figure 2). Ub acetylation negatively regulates $\mathrm{Ub}$ chain elongation by competing with ubiquitination to regulate the stability of target proteins (Ohtake et al., 2015). By using mass spectrometry (MS) approach, Ohtake et al. (2015) identified acetylation of endogenous $\mathrm{Ub}$ at residues Lys6, Lys48, and Lys63. Since the same Ub residues are involved in Ub chain formation, it is not surprising that acetylation inhibits Lys11-, Lys48-, and Lys63-linked polyUb chain elongation by several E2 enzymes in vitro, without significantly affecting E1 and E2 charging or substrate monoubiquitination (Ohtake et al., 2015). Moreover, two of the major histones, H2A and $\mathrm{H} 2 \mathrm{~B}$, were proposed as substrates for acetylated monoUb. Ser/Thr kinase PINK1 accumulates on depolarized mitochondria upon decrease in mitochondrial membrane potential and phosphorylates N-terminal Ub-like (UBL) domain of E3 ligase PARKIN (Kondapalli et al., 2012; Shiba-Fukushima et al., 2012; Kazlauskaite et al., 2014; Okatsu et al., 2018), as well as Ub itself (Kane et al., 2014; Koyano et al., 2014). Both modifications occur at the homologous position (Ser65) in PARKIN UBL domain and Ub. PARKIN UBL domain keeps PARKIN in autoinhibited state (Chaugule et al., 2011), and phosphorylated Ub is sufficient to allosterically activate it by unlocking its autoinhibition (Kane et al., 2014; Koyano et al., 2014; Wauer et al., 2015a). This topic was recently reviewed in great detail in Herhaus and Dikic (2015) and Swatek and Komander (2016). Interestingly, phosphorylation at Ser65 affects Ub structure, E2 discharging and formation of Ub chains by a subset of E2 and E3 enzymes, such as CDC34, UBC13/UEV1A, TRAF6 and HOIP (Wauer et al., 2015b). Some DUBs are also impaired in hydrolyzing Ser65-phosphoUb-containing chains (Wauer et al., 2015b). Even though other residues in $\mathrm{Ub}$ molecule have also been reported to be phosphorylated in various MS screens, the physiological significance of these modifications is not yet known.

Yang et al. (2017) have recently shown that Ub can undergo $\mathrm{NAD}^{+}$-, E1- and E2-dependent monoADP-ribosylation. The process is catalyzed by a heterodimer of ADP-ribosyltransferase PARP9 and histone E3 ligase DTX3L. Since ADP-ribose is attached to the C-terminal Gly residue of $\mathrm{Ub}$, monoADPribosylation of $\mathrm{Ub}$ prevents $\mathrm{Ub}$ conjugation and consequently impedes the Ub ligase activity and the function of DTX3L in non-homologous end joining (NHEJ) DNA repair pathway (Yang et al., 2017).

Many pathogens have evolved intricate mechanisms to hijack Ub system of the host, often mimicking components of the host Ub system, such as E3 ligases (Maculins et al., 2016) and DUBs (Pruneda et al., 2016). Recently discovered Legionella pneumophila effector SdeA utilizes unique, ATP-independent and $\mathrm{NAD}^{+}$-dependent ubiquitination mechanism that does not involve host E1 and E2 enzymes (Qiu et al., 2016). SdeA possesses intrinsic monoADP-ribosyltransferase and phosphodiesterase activities, which enable intermediate ADP-ribosylation and subsequent phosphoribosylation of $\mathrm{Ub}$ Arg40 residue. SdeA subsequently mediates ubiquitination of the target protein by conjugating phosphoribosylated $\mathrm{Ub}$ to Ser residue of the 


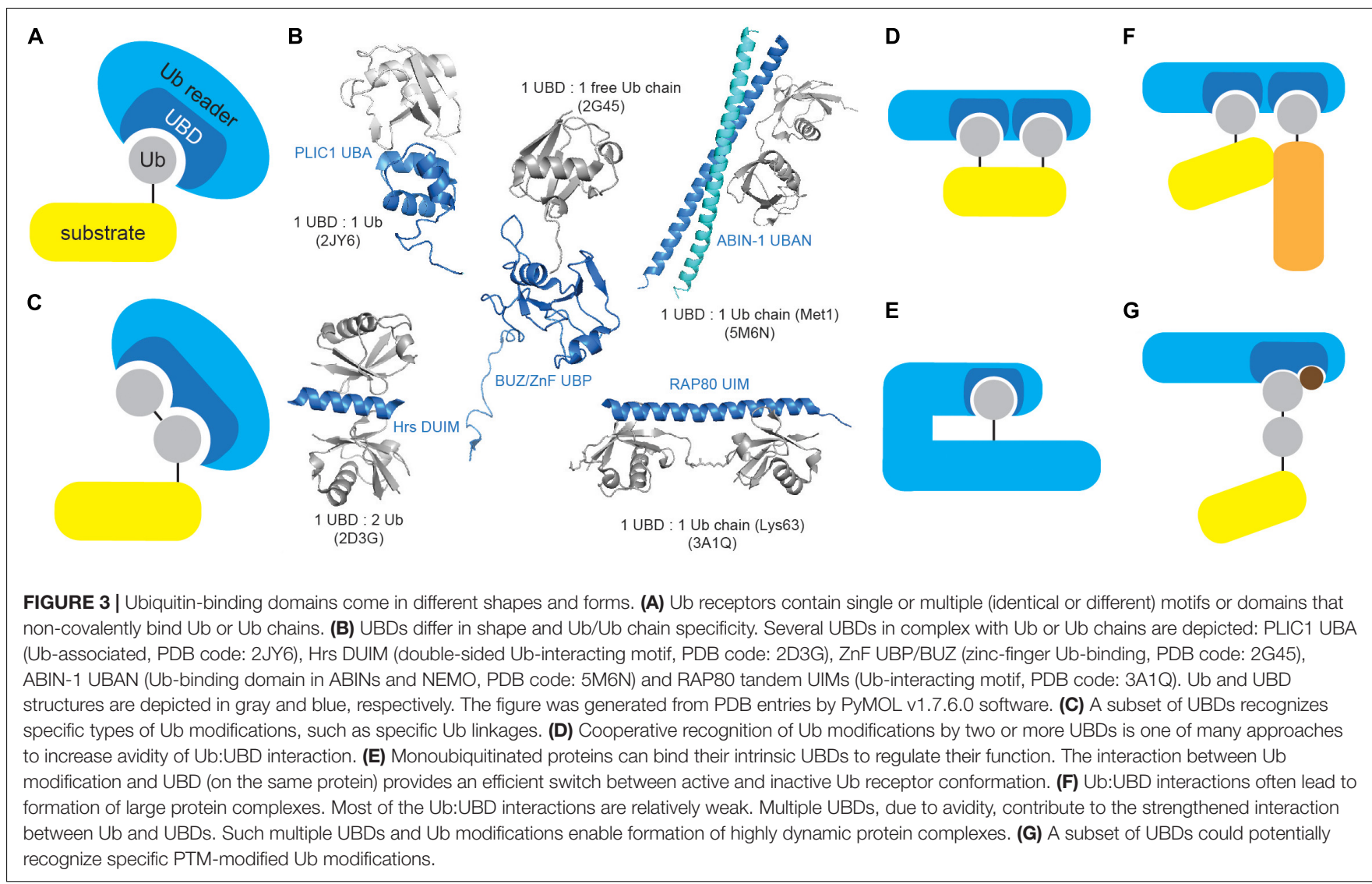

substrate through phosphodiester bond (Bhogaraju et al., 2016). Several proteins were shown to be ubiquitinated by SdeA, including small GTPase Rab33b and ER component RTN4 (Qiu et al., 2016; Kotewicz et al., 2017). Ser-linked ubiquitination of those proteins affects their cellular functions. Since SdeA-mediated ADP-ribosylation and phosphoribosylation of $\mathrm{Ub}$ inhibit activation of E1 and E2 enzymes, they also impair a plethora of essential Ub-dependent cellular processes, such as proteasomal degradation and mitophagy (Bhogaraju et al., 2016).

Cif proteins from enteropathogenic Escherichia coli (E. coli) (EPEC) and Burkholderia pseudomallei bacteria belong to the group of bacterial effectors targeting Ub signaling by catalyzing deamidatation of $\mathrm{Ub}$ at residue Gln40, which inhibits polyUb chain formation (Cui et al., 2010).

Recent proteomic studies have also revealed Ub modification by a small Ub-like modifier (SUMO) at multiple Lys residues (Galisson et al., 2011; Lamoliatte et al., 2013; Hendriks et al., 2014). Additionally, ubiquitinated SUMO has also been reported (Lamoliatte et al., 2013; Hendriks et al., 2014).

\section{Ubiquitin Readers Decode Ubiquitin Code and Induce Specific Cellular Responses}

Ub code is recognized by proteins containing single or multiple Ub-binding domains (UBDs), referred to as $\mathrm{Ub}$ readers or decoders that, more or less specifically, recognize Ub chain topology and length and enable execution of specific cellular processes (Husnjak and Dikic, 2012).

$\mathrm{Ub}$ readers interact with their targets in a transient, noncovalent way (Figure 3A) and are often found in a complex with E3 ligases and DUBs, where UBDs contribute to enzyme functionality and/or substrate selectivity, exemplified by the functional coupling between proteasomal Ub receptor RPN13 and DUB UCH37 (Reyes-Turcu and Wilkinson, 2009). Moreover, intrinsic UBDs often determine functionality of ubiquitinating and deubiquitinating enzymes.

UBDs utilize diverse surfaces to contact Ub or Ub polymers, which usually engage confined areas to interact with UBDs. Ub:UBD binding induces mild conformational changes in Ub surface, providing optimal Ub:UBD interface. Although majority of Ub surface is polar, it possesses few hydrophobic patches essential for $\mathrm{Ub}: \mathrm{UBD}$ interaction, including the most frequently utilized Ile44/Val70 patch and the less common Ile36 and Phe 4 patches (Sloper-Mould et al., 2001; Winget and Mayor, 2010). Another non-canonical hydrophobic area centered on Leu8 was identified in members of Y-family translesion synthesis (TLS) polymerases (Bienko et al., 2005). Interestingly, C-terminal part of Ub serves as a binding surface for DUB USP5/IsoT and assists in cleaving unanchored polyUb chains (Reyes-Turcu et al., 2006).

UBDs are typically independently folded, modular domains of up to 150 amino acids and with remarkable structural 
heterogeneity that can accommodate a large number of known Ub modifications. UBDs have been classified into nearly 25 subfamilies based on adapted structural folds, which can be divided into helical (i.e., Ub-associated, UBA; Ub-interacting motif, UIM), zinc finger ( $\mathrm{ZnF}), \mathrm{Ub}$-conjugating-like, pleckstrin homology $(\mathrm{PH})$ and other domains (Table $\mathbf{1}$ and Figure 3B). Interestingly, not all the members of a specific UBD family can bind Ub, as exemplified by SH3 (Stamenova et al., 2007) and CUE (Lim et al., 2019) domains.

Moreover, a subset of $\mathrm{Ub}$ readers with no obvious, structurally defined UBDs has been identified, including intrinsically disordered protein Dss1/SEM1 that binds Ub by binding sites characterized by acidic and hydrophobic residues (Paraskevopoulos et al., 2014). As such "Ubbinding activities" are hard to predict both structurally and bioinformatically, it is unclear how many of such proteins are yet to be identified.

The low-affinity interactions between UBDs and Ub are critical for rapid, timely and reversible cellular responses to a particular stimulus. However, specificity and amplification of Ub binding are required for effective and timely transmission of biological information. This is achieved by a number of different strategies. Approaches toward preferential recognition of various Ub linkages include: existence of Ub linkageselective UBDs (Figure 3C), differential $\mathrm{Ub}$ recognition by UBDs with multiple Ub-binding surfaces, $\mathrm{Ub}$ chain specificity induced by UBD dimerization or through UBD conformational adaptation, as well as through contribution of sequences situated outside UBDs to Ub binding. On the other hand, increased avidity of Ub:UBD interaction is achieved by implementing various strategies, such as: ability of UBDs to sense $\mathrm{Ub}$ chain length, cooperative $\mathrm{Ub}$ binding by tandem of identical or combination of different UBDs (Figure 3D), regulation of accessibility of Ub-binding modules (through inter- and intramolecular interactions and steric hindrance) (Figure 3E), multimerization of Ubmodified proteins and/or Ub receptors (Figure 3F) and coupled ubiquitination of UBD-containing proteins (Husnjak and Dikic, 2012; Rahighi and Dikic, 2012).

\section{Post-translational Modifications of Ubiquitin Receptors Affect Their Interactions With Ubiquitin}

Ub receptors undergo PTMs that modify their affinity to Ub (Figure 3G). Phosphorylation of selective autophagy receptor p62/SQSTM1 UBA domain (at Ser403) by casein kinase 2 (CK2) and innate immunity regulator tank-binding kinase 1 (TBK1) increases its affinity toward $\mathrm{Ub}$ and regulates autophagic clearance of ubiquitinated proteins and pathogens (Matsumoto et al., 2011; Pilli et al., 2012). Furthermore, TBK1 also phosphorylates other autophagy receptors, including OPTINEURIN (OPTN), NDP52 (CALCOCO2) and TAX1BP1 (Richter et al., 2016). Phosphorylation of OPTN UBD (Ubbinding domain in ABIN proteins and NEMO; UBAN) at residue Ser473 increases its binding capacity to various Ub chains and enables binding to Ser65-pUb chains, implicating
OPTN in PINK1-driven PARKIN-independent mitophagy (Richter et al., 2016).

\section{SENSITIVE TOOLS HELP DISSECT CELLULAR PROCESSES REGULATED BY UBIQUITIN SYSTEM}

In order to study spatiotemporal organization and dynamics of the Ub system, a set of powerful tools has been developed in the last decade, ranging from approaches that study $\mathrm{Ub}$ covalent targets, as well as non-covalent "executors" of Ub modifications. Moreover, recent advancement in techniques that enable measurement of the enzymatic activities within $\mathrm{Ub}$ system has significantly improved our understanding of the physiological significance of the Ub system (Figure 4).

\section{Methods to Study Covalent Modifications by Ubiquitin} Identification of Substrates Modified by Ubiquitin and Ubiquitin Chains

Detection and characterization of $\mathrm{Ub}$ targets are often challenging due to typically small fraction of a specific protein being modified by Ub, as well as due to highly dynamic nature of Ub modifications. Several techniques enable enrichment and identification of ubiquitinated proteins. Among those, the most common method utilizes transient or ectopic expression of $\mathrm{N}$-terminal epitope-tagged Ub variants, which can be directly conjugated to the substrates as monomers or incorporated into Lys-linked Ub polymers. The cellular epitope-tagged Ub conjugates are then enriched by affinity purification (AP) (Figure 5A). The original proteomic study identified 110 ubiquitination sites in $72 \mathrm{Ub}$ targets isolated from Ub-deficient strain of S. cerevisiae expressing 6xHIS-Ub (Peng et al., 2003). Similar strategy enabled detection of 669 Ub-modified human proteins and 44 ubiquitinated peptides in HeLa cell line (Meierhofer et al., 2008). The use of 6xHIS tag enables protein purification under denaturing conditions, thus promoting disassembly of protein complexes and inhibition of DUB activity. Due to the existence of polyHIS stretches within eukaryotic proteins, alternative tags, such as STREP, have also been developed (Danielsen et al., 2011). Another technology takes advantage of the strong biotin:avidin and biotin:neutravidin interactions and is based on the existence of biotinylatable motifs (Figure 5B) (Franco et al., 2011; Lectez et al., 2014). Here, an N-terminal, 16-amino acids biotin-accepting tag is fused to $\mathrm{Ub}$ in tandem with E. coli biotin ligase BirA. Upon biotin treatment of cells, such Ub variant can be recognized and biotinylated by BirA, followed by AP. The strategy allows in vitro and in vivo identification of high and low abundant proteins and minimization of false positive hits due to very stringent denaturing conditions (Franco et al., 2011). Proteomic analysis of in vivo biotinylated Ub enabled detection of 48 neuronal Ub conjugates from Drosophila melanogaster (D. melanogaster) embryos, as well as 393 specific ubiquitinated substrates from mouse liver (Franco et al., 2011; Lectez et al., 2014). 
TABLE 1 | List of currently known ubiquitin-binding domains.

\begin{tabular}{|c|c|c|c|}
\hline Domains & Abbreviation & Name & Examples (proteins with specific UBD) \\
\hline \multirow[t]{12}{*}{ Helical } & UIM & Ub-interacting motif & $\begin{array}{l}\text { RPN10, VPS27, USP28, ATAXIN-3, EPS15, STAM1, } \\
\text { STAM2, RAP80, DNAJB2, USP37, USP25, EPSINs }\end{array}$ \\
\hline & MIU & Motif interacting with Ub & Rabex-5, RNF168 \\
\hline & UMl & UIM- and MIU-related UBD & RNF168 \\
\hline & DUIM & Double-sided UIM & HRS \\
\hline & UBA & Ub-associated domain & $\begin{array}{l}\text { PLIC1/2, HHR23A/B, p62, NBR1, Cbl-b, USP5, } \\
\text { UBC1, HERC2, Vps13D, USP25 }\end{array}$ \\
\hline & CUE & Coupling of Ub to ER degradation domain & Cue2, Vps9 \\
\hline & GAT & GGA and TOM domain & GGA1, GGA2, GGA3, TOM1 \\
\hline & UBAN & Ub-binding domain in ABINs and NEMO & ABIN1, ABIN2, ABIN3, NEMO, OPTN \\
\hline & VHS & VPS27, HRS and STA domain & $\begin{array}{l}\text { VPS27, HRS, STAM1, STAM2, GGA1, GGA2, } \\
\text { GGA3, TOM1 }\end{array}$ \\
\hline & UBM & Ub-binding motif & Polymerase iota, Rev1 \\
\hline & MyUb & Myosin VI UBD & Myosin VI \\
\hline & AnkUBD & Ankyrin (Ank) repeat UBD & TRABID \\
\hline \multirow[t]{4}{*}{ Zinc finger (ZnF) } & UBZ & Ub-binding ZnF domain & TAX1BP1, Polymerase eta, WRNIP1, FAAP20 \\
\hline & NZF & Npl4 ZnF domain & Npl4, Vps36, TAB2, TAB3, HOIP, HOIL-1L, SHARPIN \\
\hline & $\mathrm{ZnF}$ A20 & $\mathrm{ZnF}$ of $\mathrm{A} 20$ domain & A20, Rabex-5 \\
\hline & ZnF UBP (PAZ, BUZ) & $\mathrm{ZnF}$ of Ub-specific processing protease domain & USP5, USP20, HDAC6, BRAP2 \\
\hline \multirow[t]{2}{*}{ Ub-conjugating-like } & UBC & Ub-conjugating domain & UbcH5c \\
\hline & UEV & Ub E2 variant domain & TSG101, Mms2 \\
\hline \multirow[t]{2}{*}{ Pleckstrin-homology $(\mathrm{PH})$} & GLUE & GRAM-like Ub-binding in EAP45 domain & Eap45 \\
\hline & $\mathrm{PRU}$ & Pleckstrin-like receptor for Ub & Rpn13 \\
\hline \multirow[t]{6}{*}{ Others } & & Jab1/MPN domain & Prp8 \\
\hline & PFU & PLAA family UBD & Doa1, PLAA \\
\hline & & SH3, variant & Sla1, CIN85, amphiphysin \\
\hline & & WD40 repeat $\beta$-propeller & Doa1, PLAA, Fbxw8, Met30, WDR61, PAF, WDR5 \\
\hline & & DC-UbP_N & UBTD2 \\
\hline & & MDA-9 UBD & MDA-9 \\
\hline
\end{tabular}

The observation that mutation of $\mathrm{Ub}$ residue Leu73 to Pro renders polyUb chains resistant to proteolytic cleavage by numerous DUB families led to generation of epitope-tagged Ub Leu73Pro variant that enables purification of stabilized $\mathrm{Ub}$ conjugates from cellular extracts and their subsequent proteomics-based identification (Figure 5C) (Bekes et al., 2013). Moreover, Ub and its single Lys variants, in which specific Lys residues are mutated to non-ubiquitinatable amino acids (either Arg or Ala), are frequently used to confirm ubiquitination of protein of interest and to determine the type of conjugated Ub linkage(s) (Kirisako et al., 2006; Kim and Huibregtse, 2009). Finally, since N-terminal tagging abolishes the ability of Ub to form Met1-linked (linear) Ub chains, recently developed Lysless, internally STREP II-tagged Ub (INT-Ub.7KR) has been successfully used for the MS-based AP (AP-MS) of many novel linear Ub targets (Figures 5D,E) (Kliza et al., 2017).

The abovementioned methods can be combined with either MS or traditional Western blotting.

\section{Identification of Substrates and Their Ubiquitin-Modified Sites}

Since MS enables simultaneous identification of Ub-modified proteins and precise mapping of ubiquitination sites on these proteins, several approaches were specifically designed for MS-based identification of ubiquitinated proteins. Serine protease trypsin cleaves $\mathrm{Ub}$ after residue Arg74, leaving a diGly remnant from the $\mathrm{C}$ terminus of $\mathrm{Ub}$ covalently attached to the ubiquitinated Lys residue ("Ub remnant peptide"), thus allowing localization of Ub modification ( $\mathrm{Xu}$ et al., 2010). Ub remnant profiling (Figure 6A) is a widely used immunopurification method for the identification of Lys ubiquitination sites by MS that exploits monoclonal antibody for selective enrichment of tryptic peptides containing Lys residue with diGly adduct (Xu et al., 2010; Kim et al., 2011; Wagner et al., 2011). Despite its substantial input in proteomic analysis of ubiquitinome, Ub remnant profiling has several limitations, such as additional enrichment of diGly-remnant peptides derived by tryptic digestion of UBL modifiers ISG15 and NEDD8, bias toward amino acid sequence of remnant peptides and inability to recognize linear Ub signature peptide.

Blagoy Blagoev's group has developed the StUbEx PLUS technique, which overcomes two drawbacks of Ub remnant profiling: recognition of UBL proteins and remnant peptide amino acid sequence preference (Akimov et al., 2018b). To detect ubiquitination sites, the method utilizes internally 6xHIS-tagged $\mathrm{Ub}$ in the endogenous Ub knockdown background (Akimov et al., 2018b). Insertion of 6xHIS tag near the C terminus of Ub 

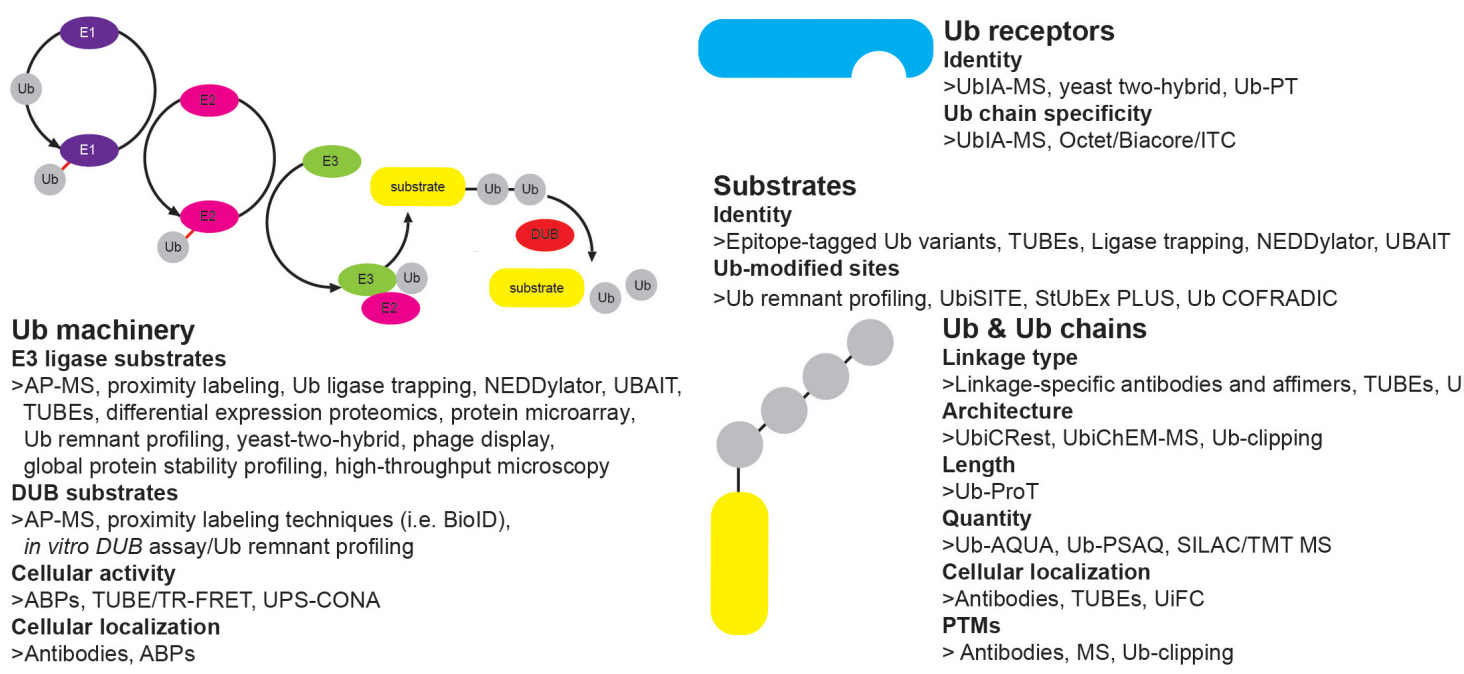

$>$ Ub remnant profiling, UbiSITE, StUbEx PLUS, Ub COFRADIC

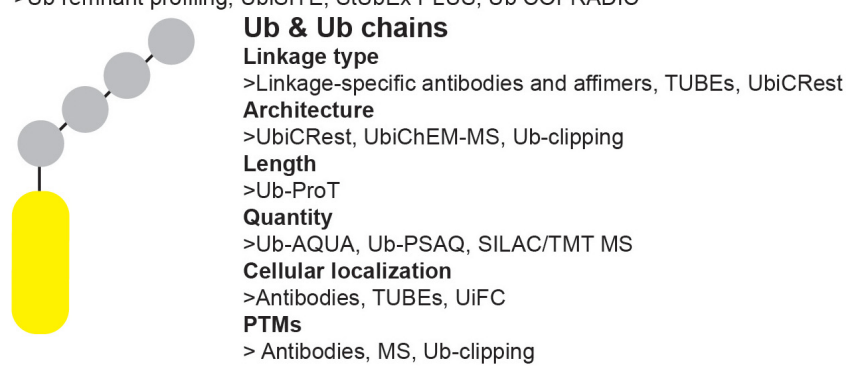

FIGURE 4 | Tools to study ubiquitin system. Overview of different approaches to study features of Ub signaling: E3 ligase and DUB enzymes (enzyme abundance, activity, cellular localization), Ub chains (type, architecture, length, quantity, cellular localization, PTMs), ubiquitinated substrates (identity, modification site, type of modification) and Ub receptors (identity, Ub linkage preference).

enables enrichment of HIS-Ub-modified substrates (Figure 5D). Subsequent proteolytic cleavage after Lys residues generates ubiquitinated peptides, which can be detected by MS. In a proof of concept experiment, StUbEx PLUS identified over 41,000 unique diGly-Ub remnant peptides in nearly 7,800 Ub targets in U2OS cells upon proteasome inhibition (Akimov et al., 2018b). However, StUbEx PLUS is more laborious technique than Ub remnant profiling.

The same group has recently developed UbiSite antibody (Figure 6B), which shows significantly improved specificity toward ubiquitinated peptides, since it recognizes the C-terminal 13 amino acids of $\mathrm{Ub}$ that remain attached to modified peptides after proteolytic digestion with the endoproteinase LysC (Akimov et al., 2018a). Importantly, the antibody also allows detection of $\mathrm{N}$-terminal ubiquitination and has enabled identification of over 63,000 unique ubiquitination sites on 9,200 proteins in two human cell lines (Akimov et al., 2018a). Nevertheless, just like Ub remnant profiling and StUbEx PLUS, it cannot recognize linear Ub signature peptide.

Noteworthy, in all of the MS-based approaches, sample preparation is critical for reliable identification of residues covalently modified by Ub. The iodoacetamide (IAA) is an alkylating chemical compound commonly used to block Cys residues in sample digestion procedures. However, IAA is not suitable for identification of ubiquitinated protein residues, as it can additionally react with unmodified Lys residues, leaving a modification of the same mass as a diGly remnant, thus mimicking ubiquitination site (Nielsen et al., 2008). Another alkylating agent, chloroacetamide, is therefore recommended for proteomic discovery of ubiquitination sites.

Combined fractional diagonal chromatography (UbCOFRADIC) is a sensitive alternative approach for identification of ubiquitination sites, initially described in Arabidopsis thaliana (A. thaliana) cells (Figure 6C). This multi-step method exploits chemical modification of free primary amines by acetyl groups, which blocks unmodified Lys residues and leaves ubiquitinated Lys residues unmodified. The subsequent deubiquitination by catalytic core of USP2 (USP2cc) exposes the now free amine groups on previously ubiquitinated Lys residues and enables the attachment of Gly-BOC tags to non-acetylated Lys residues. Trypsin digestion of such modified proteins leads to proteolytic cleavage at the C-terminus of Arg, but not Lys residues. After reverse phase HPLC, fractions containing peptides are collected and treated with trifluoroacetic acid (TFA) to remove the BOC groups. Consequently, the residues previously targeted by $\mathrm{Ub}$ are now marked by the presence of Gly residues, which can be identified by MS. This proteomics-based approach enabled identification of 3,009 ubiquitination sites on 1,607 plant proteins (Walton et al., 2016). Alike Ub remnant profiling, UbiSite and StUbEx PLUS, Ub COFRADIC is limited to MS studies and is incompatible with standard validation techniques, such as Western blotting and immunofluorescence. Noteworthy, this method generates relatively large peptides, which make MS identification more challenging.

\section{Assessment of Ubiquitin Linkage Type, Chain Size and Architecture}

Antibodies specifically recognizing $\mathrm{Ub}$ modifications are yet another type of reagents for identification of ubiquitinated proteins. While some antibodies detect all Ub-modified proteins (FK2 antibody) (Fujimuro et al., 1994), the others were engineered to selectively recognize single or a subset of specific Ub modifications, such as polyUb chain-specific FK1 antibody (Fujimuro et al., 1994). Moreover, Ub chain topology can be determined by several Ub linkage-specific antibodies, which specifically recognize Met1, Lys11, Lys27, Lys48 and Lys63 Ub linkages (Matsumoto et al., 2010, 2012; Newton et al., 2012). $\mathrm{Ub}$ antibodies have been predominantly utilized to confirm 


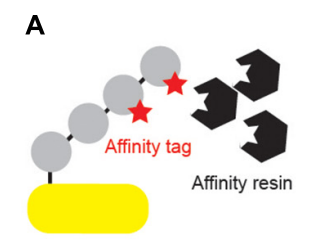

C
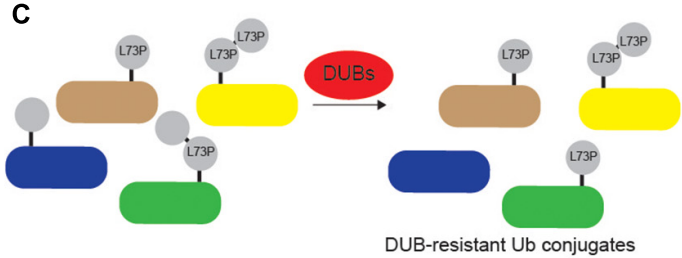

D

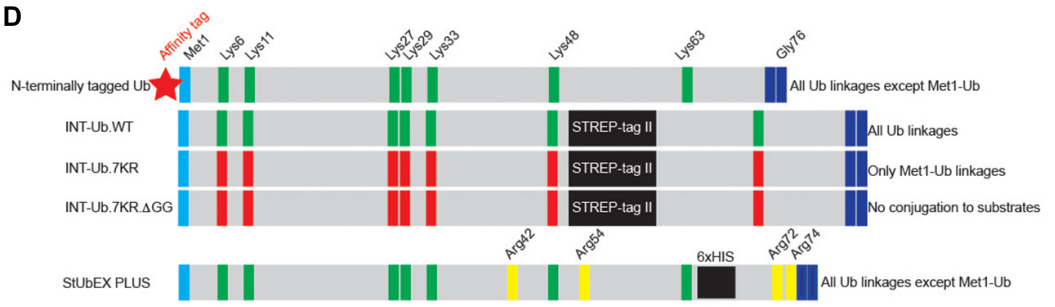

$\mathbf{E}$

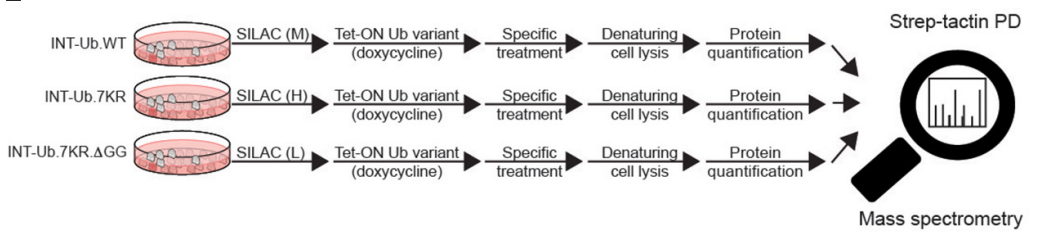

B

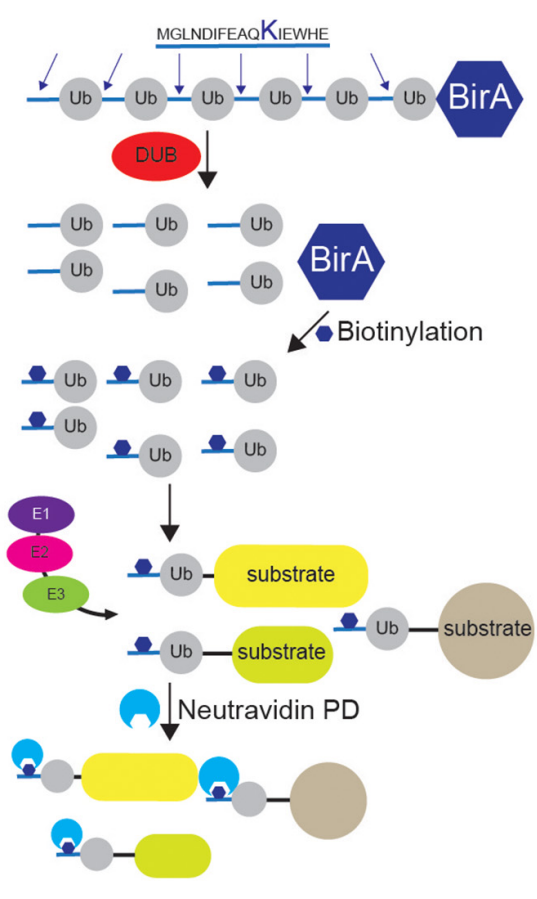

FIGURE $\mathbf{5}$ | Various approaches to study ubiquitination targets. (A) Various N-terminally tagged Ub variants can be exogenously added to cells to enable affinity purifications of ubiquitinated substrates upon denaturing lysis. Short and relatively linear tags (such as 6xHIS, STREP, HA and FLAG), combined with their respective affinity resins (Ni-NTA, strep-tactin, HA agarose and FLAG agarose) are often used in such experiments. Besides N-terminally tagged wild-type Ub, additional Ub variants are often used, such as specific Lys mutants (single or multiple Lys6, Lys11, Lys27, Lys29, Lys33, Lys48 and Lys63 residues mutated to either Arg or Ala). Additionally, residues relevant for Ub binding properties, such as lle44 and lle36, can also be mutated to either Arg or Ala. (B) Dual BirA system contains synthetic hexaUb sequence fused C-terminally to $E$. coli BirA gene ( $\mathrm{Ub}_{6}$-BirA). Each $\mathrm{Ub}$ in the construct contains 16 amino acids sequence at the $\mathrm{N}$ terminus that can be biotinylated by BirA. Once expressed in cells (or organisms), linear hexaUb is processed by cellular DUBs and undergoes biotinylation by BirA. When used by cellular ubiquitination machinery, biotin-containing Ub conjugates can be efficiently affinity purified with neutravidin resins and subsequently analyzed by Western blot and mass spectrometry. Due to the $\mathrm{N}$-terminal tagging of Ub, such approach cannot be used to enrich linear ubiquitination targets. (C) DUB-resistant Ub variant Leu73Pro increases the half-life and stability of the formed Ub linkages and facilitates their subsequent identification. (D) Two MS-coupled approaches rely on the use of internally tagged $\mathrm{Ub}$ variants. Unlike N-terminally tagged Ub, INT-Ub and INT-Ub.7KR variants enable affinity purification of ubiquitinated proteins and Met1-Ub-modified substrates, respectively, due to the existence of internal affinity purification STREP-tag between Ub residues Lys48 and Lys63 that keeps Ub Met1 free to interact with Gly76 of another Ub molecule. (E) INT-Ub approach is based on inducible expression of INT-Ub variants in SILAC-treated cells, followed by denaturing lysis, strep-tactin pull-down and subsequent MS analysis. The presence of the internal tag does not affect the overall behavior of Ub. Similar to that, 6xHIS insertion near C-terminus of Ub in StUbEx PLUS approach enables enrichment of HIS-Ub-modified substrates under denaturing conditions. The latter approach can be combined with Ub remnant profiling to identify Ub-modified substrate sites.

substrate ubiquitination and only to a lesser extent in proteomic studies (Matsumoto et al., 2010, 2012; Newton et al., 2012). The FK2 antibody was the first antibody used for initial MSbased global ubiquitination analysis, which led to identification of $670 \mathrm{Ub}$ substrates and 18 ubiquitination sites in HEK293T cells (Matsumoto et al., 2005). Similar approach resulted in detection of $70 \mathrm{Ub}$ targets from MG132-treated MCF-7 cell line (Vasilescu et al., 2005). Moreover, proteomic analysis of immunoprecipitated Met1-linkages from Salmonella-infected HCT116 cells detected 32 putative linear Ub targets (Fiskin et al., 2016). Among advantages of Ub antibodies are detection of ubiquitinated proteins at the endogenous level and a wide applicability, including Western blotting, immunoprecipitation, immunofluorescence and flow cytometry. Since Ub linkagespecific antibodies often exhibit high cross-reactivity, their usage requires proper controls and highly defined experimental conditions (Beaudette et al., 2016).
Yet another technique for identification of Ub-modified proteins and evaluation of their Ub modifications is based on Ub-binding modules. Due to typically low binding affinity of UBDs toward Ub, synthetic multiple repeats of UBDs (tandem Ub-binding entities, TUBEs) were engineered (Figure 7A). Expressed as recombinant epitope-tagged fusions, those tools are characterized by high overall Ub-binding avidity and enable efficient capturing of both high and low abundant Ub targets from cellular lysates (Hjerpe et al., 2009; Mattern et al., 2019). A large number of available Ub traps differs in number and types of UBDs, length of linkers and type of epitope tags. While affinity UBD-based tools are widely used for the confirmation of specific protein ubiquitination, several reports demonstrated their applicability for proteomic analysis of ubiquitinated proteins. Identification of over 290 ubiquitination sites and 223 putative ubiquitinated proteins in HEK293T cells has been demonstrated in ubiquitinome 

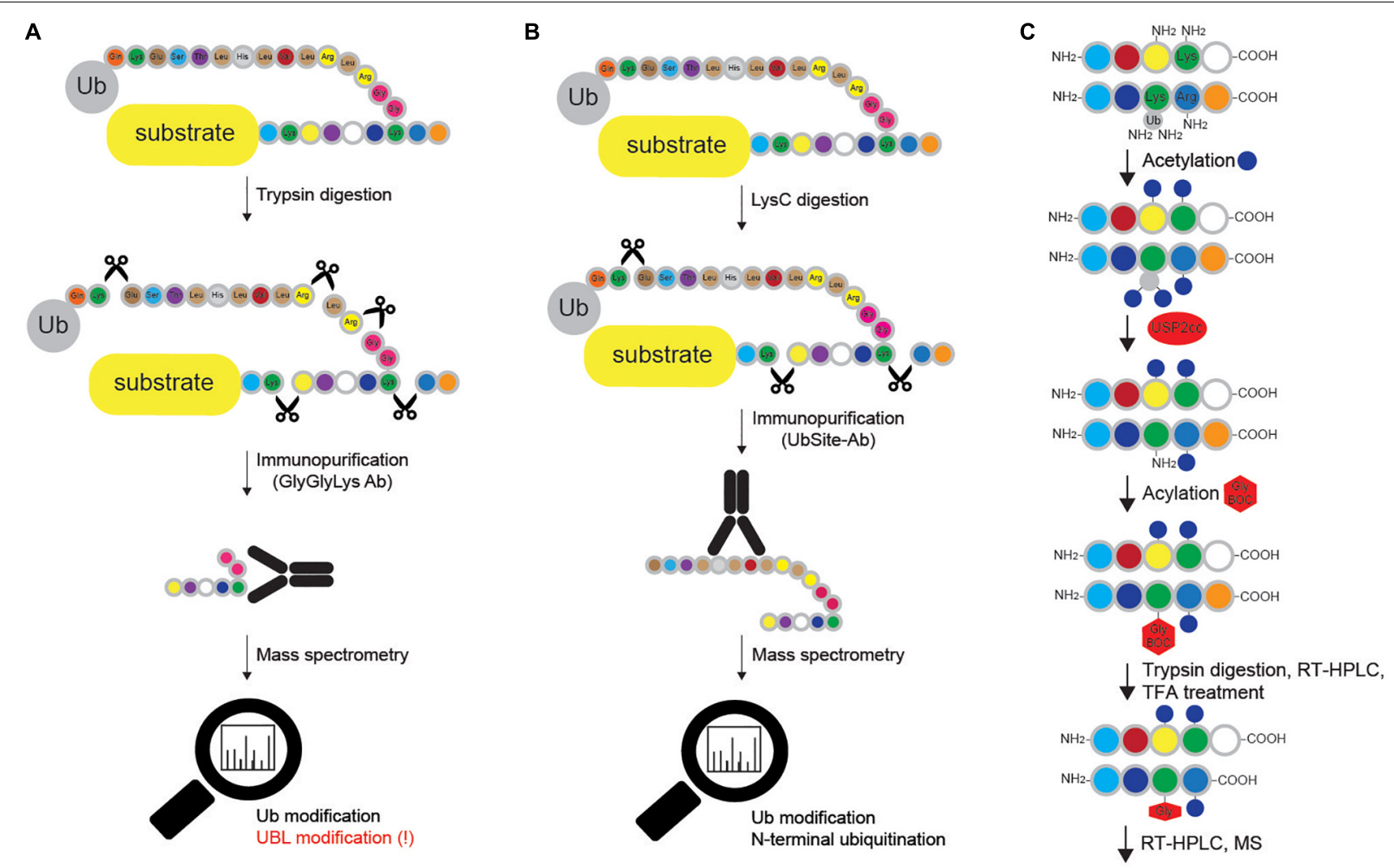

FIGURE 6 | Methods for mapping ubiquitination sites in proteins. (A) Ub remnant profiling is based on trypsin digestion of the proteome (cells are previously lysed in urea-containing buffer) combined with immunoprecipitation with monoclonal antibody raised against Lys- $\varepsilon$-Gly-Gly motif that remains on ubiquitinated substrate after trypsin cleavage. Samples are further processed and analyzed by LC-MS/MS. Such approach does not distinguish between modifications by Ub and other UBLS (such as NEDD8 and ISG15), and cannot be applied for Met1- and N-terminally Ub-modified proteins. (B) UbiSite antibody recognizes the last 13 amino acids of Ub that remain attached to ubiquitinated proteins upon LysC cleavage. Enriched ubiquitinated proteins are further analyzed by MS. Even though UbiSite approach distinguishes between modifications by Ub and other UBLs, it cannot be used for studying linear ubiquitination, as it does not recognize the signature peptide of linear ubiquitination after tryptic cleavage: Gly-Gly-Met-Gln-Ile-Phe-Val-Lys. (C) Ub-COFRADIC approach distinguishes between free ( $\alpha$ or $\varepsilon$ ) and modified primary amines to enable identification of ubiquitinated Lys residues. Initial acetylation by NHS-acetate is only possible on free amines, leaving ubiquitinated Lys residues non-acetylated. Subsequent addition of USP2cc removes all the Ub moieties from Lys residues and enables the attachment of Gly linked to a hydrophobic tert-butyloxycarbonyl (Gly-BOC tag) to previously non-acetylated Lys residues. Trypsin then cleaves C-terminally of Arg residues (but not C-terminally of acetylated Lys). Peptides collected after the first reversed phase (RP)-HPLC run are treated with TFA to remove BOC groups, followed by additional RP-HPLC and MS. In enzyme setting during MS data analysis, ArgC (and not trypsin) should be selected, as cleavage after Lys residues is blocked.

analysis, which used PLIC-1 UBA-based TUBE (Shi et al., 2011). Another proteomic study utilized a tandem of hybrid UBDs (ThUBDs) to analyze total ubiquitinated proteins, which enabled detection of 1092 and $7487 \mathrm{Ub}$ targets in yeast and liver MHCC97-H cell line, respectively (Gao et al., 2016). The recombinant HIS fusion of PLIC2 UBA domain enabled enrichment of polyUb chains from brains of Huntington's disease model mice, as well as patient samples (Bennett et al., 2007). Moreover, Ub linkage-selective affinity UBDbased probes have also been developed, including Lys29/Lys33 linkage-specific (TRABID NZF-based), Lys63 linkage-specific (TAB2 NZF- and VPS27 UIM-based) and Met1-linkage-specific (UBAN-based) TUBEs (Emmerich and Cohen, 2015). By using tandem VPS27 UIM-based probe, over 100 putative Lys63 Ub-modified proteins were identified in A. thaliana (Johnson and Vert, 2016). UBAN-based M1-SUB probe combined with proteomic analysis identified a single linear Ub-modified substrate in THP-1 cells upon NOD2 stimulation (Fiil et al., 2013). Furthermore, the tandem of $\mathrm{ZnF}$ UBP domain and hybrid Ub probe comprised of ZnF UBP and UBA domains were designed for isolation of unanchored $\mathrm{Ub}$ chains and unconjugated Lys48 linkages, respectively (Scott et al., 2016). To summarize, affinity UBD-based reagents efficiently enrich ubiquitinated endogenous substrates, protect Ub conjugates from DUB-mediated proteolysis and proteasomal degradation and have a wide range of applications, including MS, Western blotting and microscopy (Hjerpe et al., 2009; Shi et al., 2011; van Wijk et al., 2012). However, the use of UBD-based tools for discovery of ubiquitinated substrates requires non-stringent purification conditions that ultimately lead to purification of protein complexes rather than individual Ub-modified proteins. On top of that, some UBDs also bind proteins containing intrinsic UBL domains, which results in a relatively high number of contaminants. 


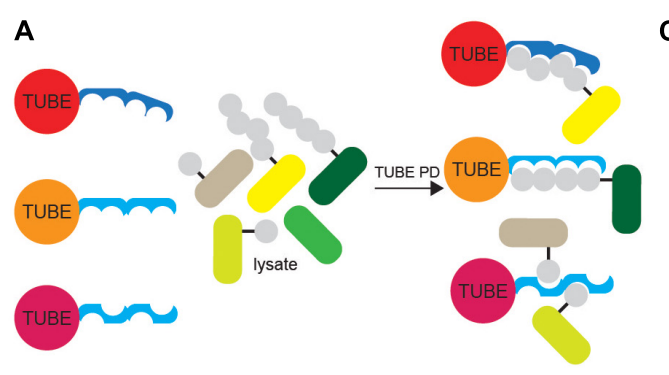

C

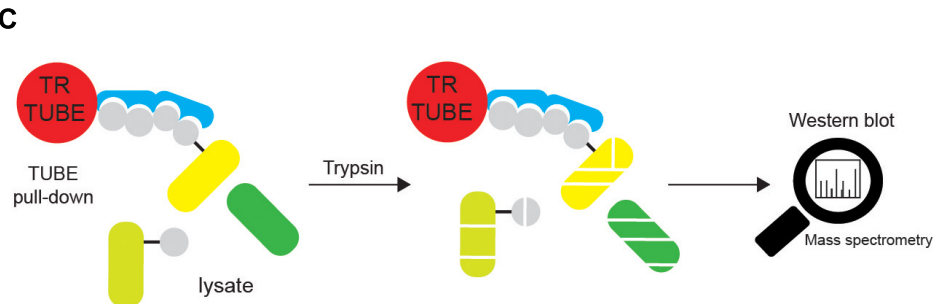

B

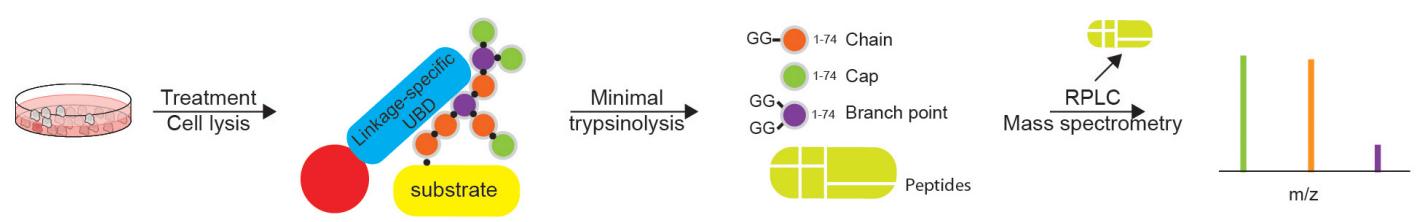

FIGURE 7 | Tandems of ubiquitin entities can be utilized in various ubiquitin tools. (A) Tandems of Ub chain-specific or promiscuous UBDs, additionally equipped with affinity tags (such as FLAG-, HA-, GST-, or 6xHIS) and bound to appropriate resins, can be used for affinity purification of ubiquitinated proteins. The use of tandem UBDs increases affinity toward Ub due to avidity, as well as protects ubiquitinated proteins from endogenous DUBs during purification steps. Purified ubiquitinated proteins can be further analyzed by either Western blotting or MS. (B) Ub chain enrichment middle-down MS (UbiChEM-MS) approach is based on the enrichment of the specific Ub linkages by linkage-specific UBDs or antibodies, combined with minimal trypsinolysis of Ub that induces a single Ub cleavage after Arg74 and leaves the rest of the Ub molecule intact. By using that approach, Ub molecules within chain, as well as capping and branched Ub conjugates can be detected and quantified by MS. (C) Ub-ProT (Ub chain protection from trypsinization) method determines the length of Ub chains bound to target proteins. Trypsin-resistant (TR)-TUBE (i.e., PLIC1 UBA domain lacking Arg residues) is used for the enrichment of ubiquitinated proteins that can be analyzed by Western blotting. Furthermore, since TR-TUBE-protected sample is resistant to trypsin digestion, it can be applied for the determination of the length of Ub chains by quantitative MS.

Since neither antibodies nor TUBEs selectively recognizing several atypical Ub linkages were available, David Komander's group used the affimer technology to screen libraries of small, non-antibody protein scaffolds with randomized surface to develop the linkage-specific Ub affinity reagents for detection of Lys6- and Lys33-linked polyUb chains (Michel et al., 2017). The Lys6 affimer exhibits high selectivity toward Lys6-linked polyUb chains, whereas the Lys33 Ub affinity reagent also recognizes Lys11 linkages. The proteomic analysis of proteins enriched by Lys6 Ub affimer enabled identification of mitofusin2 and HUWE1 as the Lys6 polyUb-specific substrate and E3 ligase, respectively. Both linkage-specific Ub affinity reagents are suitable for in vitro and in vivo binding assays, MS, Western blotting and immunofluorescence.

Ub Chain Restriction (UbiCRest) approach is mainly utilized to confirm ubiquitination of putative substrates, which were identified by other methods (Hospenthal et al., 2015). UbiCRest kit provides a set of recombinant DUBs of defined linkage specificities that enable qualitative determination of the type(s) and architecture of Ub linkages modifying protein of interest (Hospenthal et al., 2015). Hitherto, several studies successfully applied UbiCRest, as shown by validation of linear polyUb targets BCL10, CASP8 and TNFR1 (Satpathy et al., 2015; Emmerich et al., 2016; Lafont et al., 2017). However, obtained results highly depend on numerous factors, including reaction conditions, concentration and enzymatic activity of DUBs, incubation period, as well as method used for the enrichment of Ub-modified substrate (Hospenthal et al., 2015).

Although linkage-specific antibodies can be used for studying endogenous polyUb-modified proteins, they cannot clearly distinguish between homotypic and heterotypic Ub chains. Bispecific antibodies detecting heterotypic Ub chains exist so far only for Lys11/LysK48-linked Ub chains (Yau et al., 2017). Complex topology of Ub chains, including branched Ub linkages, prompted the development of novel MS-based approaches for simultaneous detection of multiple modifications on a single Ub moiety. While bottom-up MS (such as Ub remnant profiling) enables characterization of linkages between two Ub molecules, it cannot assess chain length and topology due to trypsin digestion. Opposite to that, middle-down MS utilizes minimal protease digestion of protein samples to detect multiple PTMs on a single Ub molecule (Figure 7B). It is based on the notion that under optimized conditions, native folded polyUb is trypsinized only at the Arg74 residue (Xu and Peng, 2008). In that way, minimal trypsinolysis, by leaving Ub largely intact, enables detection of multiple modifications by MS (Xu and Peng, 2008; Valkevich et al., 2014). Ub chain enrichment middle-down MS (UbiChEM-MS) approach combines the enrichment of specific Ub chains using linkage-specific UBDs with minimal trypsinolysis and middledown MS for the characterization of branched Ub conjugates (Crowe et al., 2017).

Furthermore, David Komander's group has recently published Ub-clipping approach that utilizes an engineered viral protease $\left(\mathrm{Lb}^{\text {pro*}}\right)$ to incompletely remove Ub from substrates, leaving the C-terminal diGly dipeptide conjugated to the modification site and enabling quantification of multiply diGly-modified branchpoint Ub (Swatek et al., 2019). By using that approach they could estimate that around $10-20 \%$ of Ub in polymers can be found in branched Ub chains. 
The length of substrate-attached Ub chains is usually estimated by monitoring their gel mobility in SDS-PAGE. However, due to the complex nature of ubiquitination (different Ub modifications can be simultaneously attached to a single protein), determining the length of $\mathrm{Ub}$ chains is not straightforward. Ub-ProT (Ub chain protection from trypsinization) method (Figure 7C) was recently developed for assessing the length of substrate-attached polyUb chains (Tsuchiya et al., 2018). The method is based on the use of Ub chain protector, i.e. trypsin-resistant (TR)-TUBE, which consists of biotin and 6xHIS tags and six tandem repeats of the PLIC1 UBA domain, in which all the Arg residues are replaced by Ala (to prevent trypsin digestion of the TUBE). When substrateattached Ub chains are bound by TR-TUBE, they are resistant to trypsin digestion and can be analyzed using a gel-based assay. By combining this method with quantitative MS analysis, Tsuchiya et al. (2018) determined the length and composition of Ub chains in yeast, and of ligand-activated epidermal growth factor receptor (EGFR) in mammalian cells. The observed disadvantage of the Ub-ProT approach is differential protection of various Ub linkages, as some of them (such as Lys6, Lys27, Lys29, and Lys33) are less efficiently protected, similar to decreased protection of branched over homotypic Ub linkages, which will inevitably generate bias in data analysis. The development of novel TR-TUBEs, with a uniform affinity toward all Ub linkages and without preference for homotypic over heterotypic/branched Ub linkages should improve the quality of this approach.

\section{Quantification of Ub Modification}

In general, quantitative measurements of ubiquitination can be either relative or absolute and involve the use of various labeling approaches, such as metabolic labeling (stable isotope labeling with amino acids in cell culture; SILAC) or isobaric peptide tagging (isobaric tags for relative and absolute quantitation; iTRAQ and tandem mass tags; TMT).

Absolute quantification (AQUA) strategy is often used for absolute quantification of proteins or PTMs. Isotopically labeled synthetic peptide, corresponding to the tryptic peptide of the protein of interest, is used as an internal standard with a known concentration. In the Ub-AQUA approach, all eight ubiquitinated diGly peptides can be labeled with a stable isotope and used as internal standard that can be readily distinguished by MS and used for quantification of corresponding native peptides (Figure 8A) (Kirkpatrick et al., 2005). For examples, Ub-AQUA approach was used to quantify various types of Ub modifications of NEMO, an essential regulator of NFKB signaling (Ikeda et al., 2011).

Since Ub-AQUA approach cannot take into account any experimental loss of protein, Ub-PSAQ approach has additionally been developed. MS-based Ub protein standard absolute quantification (Ub-PSAQ) approach uses stable isotope-labeled free $\mathrm{Ub}$ and $\mathrm{Ub}$ conjugates as recovery standards, which are added into lysates and captured with affinity reagents either selective for free Ub ( $\mathrm{ZnF}$ UBP domain that captures unconjugated $\mathrm{Ub}$ by interacting with $\mathrm{C}$ terminus of $\mathrm{Ub}$ ) or $\mathrm{Ub}$ chains (PLIC2 UBA domain that binds Ub chains) (Figure 8B). Additionally, half of the sample is treated with USP2cc, which enables the conversion of all $\mathrm{Ub}$ species to free $\mathrm{Ub}$ in order to measure total Ub, and captured by UBD ZnF UBP/BUZ affinity reagent. Sample is subsequently washed, eluted, treated with trypsin and quantified by LC-ESI TOF MS relative to the peptide standard. The presence of DUB inhibitor in the assay prevents interconversion of Ub species during assay (Kaiser et al., 2011).

\section{Determination of Cellular Localization of Ubiquitin Modifications}

The techniques and approaches for visualizing Ub signals have recently been reviewed in details by van Wijk et al. (2019).

Engineered UBD-based biosensors containing fluorescent tags found important applications for Ub-binding modules in in vivo visualization of Ub modifications. The UBAN-based biosensor enabled monitoring of Met1 linkages in TNF $\alpha$-mediated NFKB signaling and co-localization of linear Ub chains with cytosolic Salmonella during xenophagy, whereas Lys63-selective UIMand NZF-based sensors traced localization and accumulation of Lys63 linkages during DNA damage response (DDR), mitophagy and upon IL-1 $\beta$ and TNF-related weak inducer of apoptosis (TWEAK) stimulation (Sims et al., 2012; van Wijk et al., 2012).

Ubiquitination-induced fluorescence complementation (UiFC) assay is a variation of TUBE-based biosensors. In this technique, visualization of polyUb chains is achieved by expression of two non-fluorescent, complementary fragments of a fluorescent protein fused to UBDs. Upon UBD-mediated binding to polyUb chains in close proximity, the fluorescence of two fragments is restored (Chen et al., 2013; Pinto et al., 2016). As a proof of concept, Lys48-linked Ub chains were visualized with Epsin1 UIM-based UiFC biosensors under various conditions (i.e., mitophagy, proteasome inhibition) (Chen et al., 2013). Importantly, levels of UBD-based biosensors have to be kept low during experiment, as their high expression could restrict activation of cellular signaling pathways (Sims et al., 2012; van Wijk et al., 2012).

Several aforementioned Ub and Ub linkage-specific antibodies (i.e., FK1 and FK2, Lys48- and Lys63-linked polyUb) are suitable for immunocyto- and immunohistochemistry and are widely used (Newton et al., 2008; Danielson and Hope, 2013; Nakazawa et al., 2016). Among others, Met1-linked polyUbspecific antibody was used to determine the effect of linear Ub binding-deficient OPTN mutations in the onset of amyotrophic lateral sclerosis (Nakazawa et al., 2016).

\section{Identification of Post-translationally Modified Ubiquitin and Ubiquitin Chains}

Discovery of PTMs that modify Ub monomers and polymers has predominantly been achieved by MS. As delineated previously, MS detection of tryptic Ub peptides containing specific modifications enabled identification of Ub PTMs such as deamidation and phosphoribosylation (Cui et al., 2010; Bhogaraju et al., 2019).

The abovementioned Ub-clipping method (Swatek et al., 2019) can also be used to determine the co-existing PTMs on Ub modifications. As a proof of principle, such approach was used to determine Ub architecture on depolarized mitochondria. The analysis revealed that, under mitophagy-inducing conditions, 
A
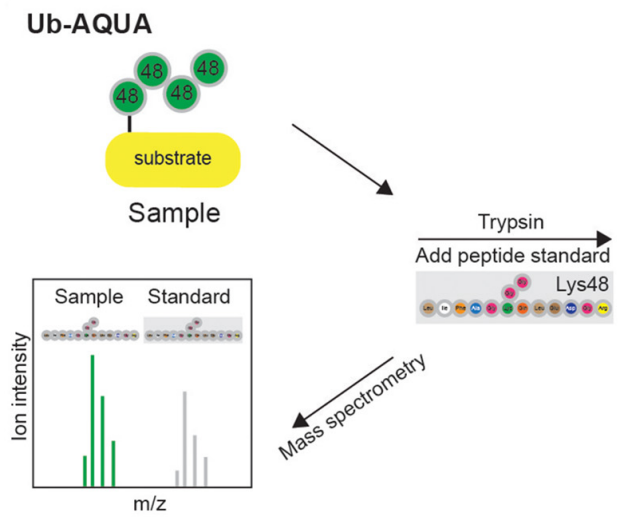

B
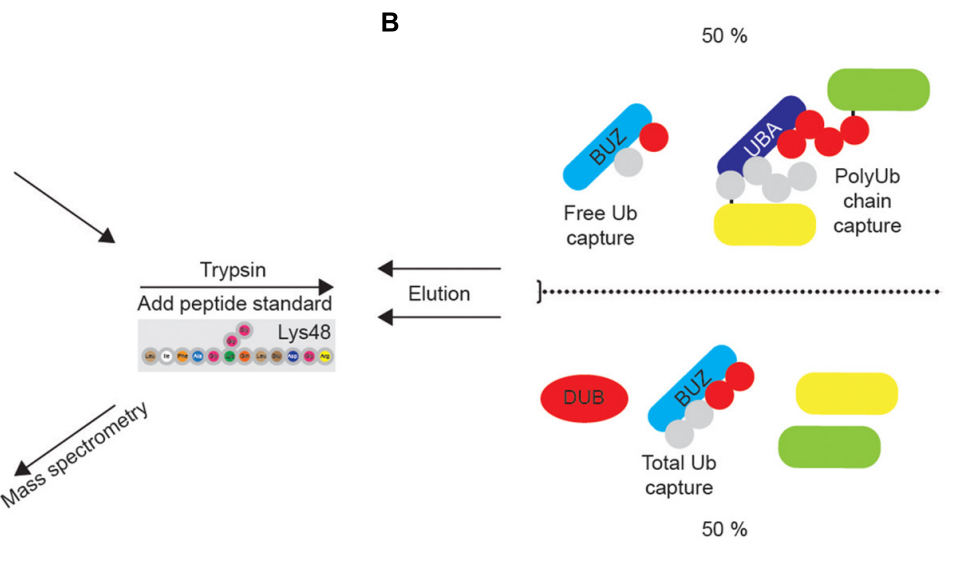

$50 \%$
Ub-PSAQ

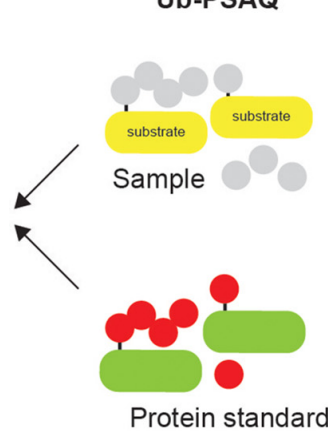

FIGURE 8 | Quantification of ubiquitination in vivo. (A) Synthetic peptide absolute quantification (Ub-AQUA) MS approach is based on synthetic peptides as quantification standards for both mono- and polyubiquitination. (B) Ub-PSAQ approach is based on the use of stable isotope-labeled free Ub and Ub conjugates as protein standards. They are added to lysates and captured with UBD BUZ that is selective for free Ub and UBD PLIC2 UBA (or similar) that recognizes Ub chains. Half of the sample is treated with USP2cc and total free Ub captured by BUZ affinity reagent. Sample is quantified by MS, relative to the peptide standard.

$\mathrm{Ub}$ coat on mitochondria is composed mainly of monoUb and oligoUb chains, with phosphoUb capping Ub chains and therefore, preventing further extension of Ub polymers (Swatek et al., 2019).

Currently, there are several available antibodies that detect specific PTMs on Ub. The antibody recognizing phosphorylation on Ub Ser65 is suitable for Western blotting and has been used to study the effect of Ub phosphorylation on the recruitment of Ub-binding mitophagy receptors to depolarized mitochondria (Ordureau et al., 2018). Moreover, the antibody detecting acetylation on $\mathrm{Ub}$ Lys48 residue, suitable for ELISA and Western blotting, is available on the market. However, it has not been reported in any publication thus far.

\section{Methods to Study Ubiquitination Machinery Identification of E3 Ligase Substrates}

Identification of E3 ligase:substrate pairs is very challenging, since interactions between E3 ligases and their targets are very dynamic and of low affinity. Moreover, ubiquitination of the substrates often exhibits stimulus- and spatiotemporal dependency. Furthermore, individual substrates can be targeted by several E3 ligases at different residues and at different physiological conditions. Additionally, ubiquitinated substrates are often marked for proteasomal degradation, which leads to their fast removal from the cells.

A plethora of approaches has been established to enable identification of E3 ligase substrates and review by Iconomou and Saunders discusses them in details (Iconomou and Saunders, 2016). These include proximity-dependent biotin labeling (BioID) (Roux et al., 2012; Coyaud et al., 2015), Ub ligase substrate trapping (Mark et al., 2014, 2016; Loveless et al., 2015), Ub-activated interaction traps (UBAIT) (O'Connor et al., 2015, 2018) and NEDDylator approach (Zhuang et al., 2013).
BioID approach allows the identification of proteins in the close vicinity of a protein of interest in living cells. It is based on the fusion of the E3 ligase with mutated form of biotin ligase BirA, which biotinylates all the proteins in the close vicinity (around $10 \mathrm{~nm}$ ), if biotin is available. Such "neighborhood tagging" allows AP and subsequent MS-based detection of all the labeled proteins, majority of which potentially being the E3 ligase substrates. This approach enabled identification of 50 putative substrates of $\mathrm{SCF}^{\beta \mathrm{TrCP} 1 / 2}$ (Coyaud et al., 2015).

Ligase trapping is an AP approach in which E3 ligases fused to UBDs are used for isolation of ubiquitinated substrates (Figure 9A). The presence of UBD increases the binding affinity of the E3 ligase of interest toward its targets, thus increasing sensitivity of the method. This technique enabled successful identification of novel substrates of FBXL E3 ligases, including Prb1 (Mark et al., 2014). The selection of proper UBD (to ensure effective enrichment of substrates), as well as fusion point (that might potentially disrupt the substrate recruitment) is essential for the proper functionality of the ligase trap.

UBAIT is a method belonging to ligase trapping class and allows for identification of substrates for HECT and RING E3 ligases (Figure 9B). UBAIT tool consists of E3 ligase fused to $\mathrm{Ub}$ moiety and target-interacting domain. The presence of Ub enables E1- and E2-mediated activation of UBAIT and subsequent covalent capture of E3 ligase substrates. This technique was applied to identify proteins interacting with several Ub ligases, such as ITCH and RNF126 (O'Connor et al., 2015). The drawback of this approach is that it cannot distinguish between E3 ligase substrates and E3 ligaseinteracting proteins.

NEDDylator approach relies on the fusion between NEDD8 E2 enzyme and substrate-binding region of desired E3 ligase (Figure 9C). Such configuration allows artificial NEDDylation of endogenous E3 ligase substrates, their enrichment (by denaturing immunoprecipitation of exogenous NEDD8 tag, such as 6xHIS) and subsequent MS identification. As NEDDylation does not 

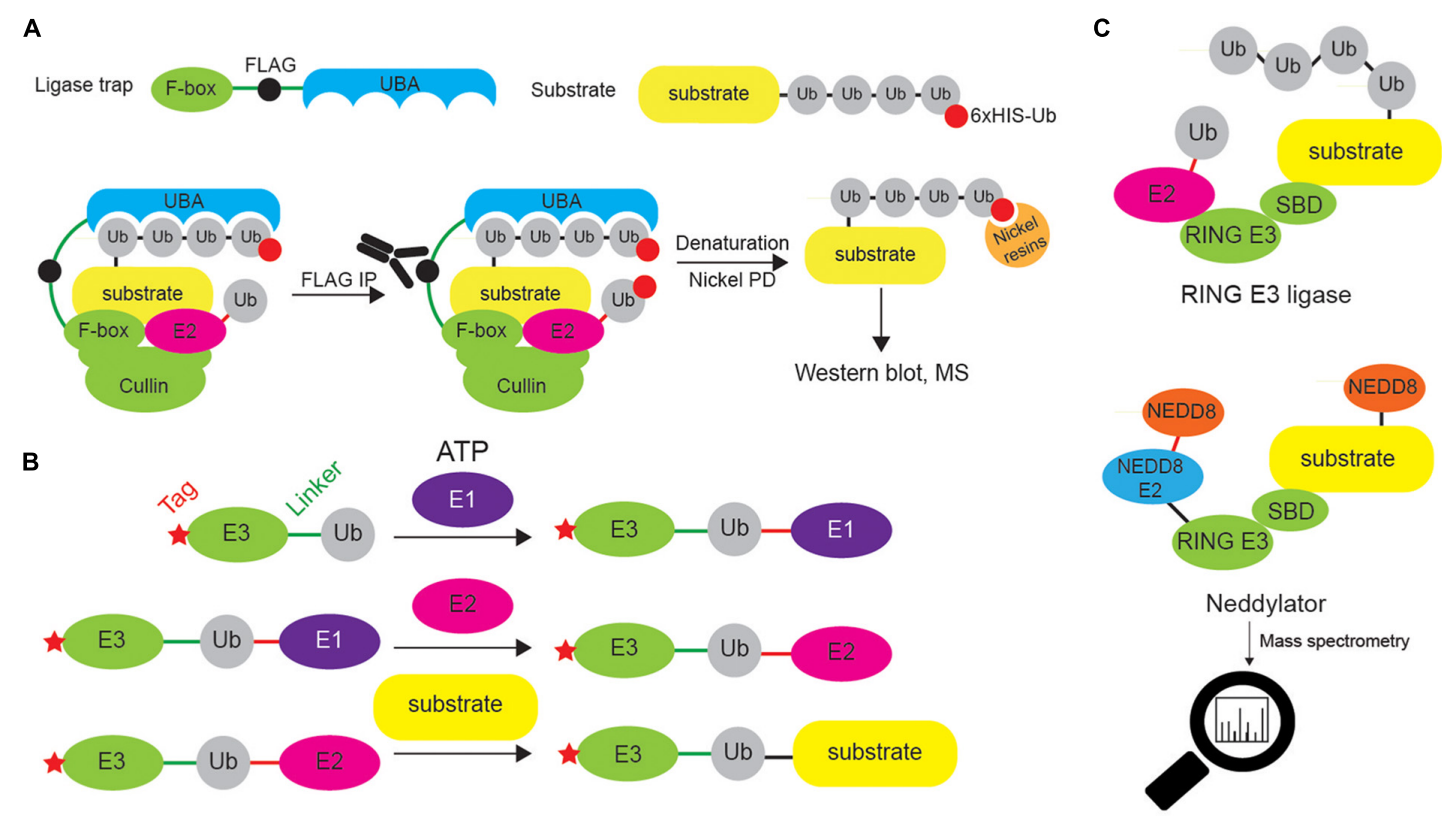

FIGURE 9 | Different approaches to identify E3 ligase substrates. (A) Ligase trapping approach "stabilizes" E3 ligase:substrate non-covalent interactions by UBDs (such as UBA domain) fused to E3 ligase substrate-interacting domains (such as F-box of the multi-protein E3 ligase complex SCF). When such ligase traps and 6xHIS-tagged Ub are overexpressed in cells, the UBA interacts with the nascent Ub chain on endogenous SCF substrates, thereby delaying their release. Cells are then lysed and subjected to an anti-FLAG coimmunoprecipitation under native conditions, to isolate ligase trap complexes (FLAG tag is inserted between F-box and UBA). FLAG eluates are then used in denaturing Ni-NTA agarose pull-down to exclusively enrich ubiquitinated substrates (and to remove any non-covalently interacting proteins). (B) UBAITs, similar to ligase traps, enable identification of E3 ligase substrates (for both HECT and RING E3 ligases), as well as their adaptors and regulators. Unlike ligase traps, UBAITs are fusions of N-terminal affinity-tagged E3 and C-terminal Ub molecule. With the help of cellular E1 and E2, UBAIT E3 component transfers UBAIT Ub component (by forming amide bond) to proteins that interact with the E3, such as E3 ligase substrates. Formed complex is easily affinity purified and analyzed by mass spectrometry. For HECT E3s, both E3 and E2 thioester-linked interacting proteins can be captured by UBAITs. (C) NEDDylator is a catalytic tagging tool, in which Ubc12, an E2 enzyme for NEDD8, is fused to an E3 ligase substrate-binding domain, allowing for the transfer of NEDD8 to the E3 substrate, and MS-based identification of E3 ligase-target pairs.

occur at a high level in a cell, it is not difficult to distinguish between endogenous and NEDDylator-induced modifications and as such, to identify E3 ligase substrates.

\section{Identification of DUB Substrates}

Identification of DUBs and their targets is difficult due to several reasons: the enzymatic activity of DUBs results in a rapid removal of Ub modifications from the DUB substrates, the interaction between DUBs and their substrates is often inducible and spatiotemporally restricted, DUBs typically bind their substrates with relatively low affinity and numerous DUBs require accessory proteins for specific interactions with their targets. Therefore, a limited number of studies have aimed to identify the substrates for specific DUBs thus far.

A common technique is substrate AP with either recombinant or ectopically expressed epitope-tagged DUB as bait (Bonacci et al., 2018). However, this approach preferentially identifies DUB interactors over DUB substrates. If known, point mutation of the active site of investigated DUB, which decreases/abolishes the proteolytic activity of the DUB, greatly facilitates identification of DUB targets as it enables entrapment of ubiquitinated substrates. This approach led to the identification of APC/C substrates (i.e., Cyclin B and Aurora A) as targets of Cezanne/OTUD7B, a Lys11 linkage-specific DUB (Bonacci et al., 2018). Another study utilized similar approach to identify NFX1-123 as a substrate of USP9X (Chen et al., 2019).

Yet another method for discovery of DUB targets is based on in vitro deubiquitination of cell lysate with recombinant DUB of interest. Together with reference lysate, samples are then digested with trypsin, peptides are isotopically labeled and subjected to Ub remnant profiling. This quantitative proteomic approach enabled identification of two substrates of Salmonella Typhimurium effector SseL (Nakayasu et al., 2015).

\section{Measuring the Enzymatic Activity of Ubiquitination Machinery}

E3 ligases and DUBs have evolved in the last several years as promising therapeutic targets in oncology and neurodegeneration, as they are often perturbed in various diseases and cancer types (Cromm and Crews, 2017; Harrigan et al., 2018; Yang et al., 2018). Many research groups and pharmaceutical industry are therefore developing specific inhibitors and activators of these enzymes, as well as improving and developing quantitative methods for measuring their enzymatic activity both in vitro and in vivo. A comprehensive review about activity-based probes (APBs) for ubiquitination machinery has recently been published by Huib Ovaa's group (Witting et al., 2017). 
Initially developed ABPs contain Ub moiety with the C-terminal Gly76 residue chemically modified with an electrophilic warhead, such as aldehyde, vinyl sulfone (UbVS), vinyl methylester (Ub-VME) and propargylamide (Ub-Prg) to covalently bind proteins containing active Cys residue (Borodovsky et al., 2001, 2002; Ekkebus et al., 2013). They have been successfully used to identify novel DUBs and monitor DUB activity (Borodovsky et al., 2002; de Jong et al., 2012). The real advancement in the field came after the successful diUb chemical synthesis, which opened new possibilities in developing DUB probes (El Oualid et al., 2010; de Jong et al., 2012; Mulder et al., 2014; Flierman et al., 2016). Many approaches have also been developed for assessing DUB specificity, ranging from diUb probes mimicking all eight different Ub linkages combined with MS (McGouran et al., 2013), diUb probes resembling native diUb that contain a Michael addition acceptor for trapping the DUB active-site Cys (Li et al., 2014), seven synthetic isopeptide-linked diUb FRET probes with rhodamine-TAMRA for the absolute quantification of chain cleavage specificity (Geurink et al., 2016) or monitoring total cellular DUB activity by advanced chemoproteomics (Pinto-Fernandez et al., 2019).

Many assays for assessment of E3 ligase activity are based on monitoring E3 ligase autoubiquitination, either by Western blotting or by measuring fluorescence. By combining timeresolved fluorescence resonance energy transfer (TR-FRET) based on lanthanide chemistry and TUBEs, Marblestone et al. (2012) have developed an E3 ligase activity assay in which they monitored the proximity of the autoubiquitinated E3 ligase and biotinylated TUBEs in an E3-dependent polyUb chain formation assay based on endogenous Ub. The method is limited to studying E3 ligase activity of the specific, individual E3 ligase in vitro and might not work in cellular lysates. For E3 ligases that require
PTMs or additional protein components for their enzymatic activity such approach can be technically very challenging.

The development of E1-E2-E3 activity probes was not as fast as the development of DUB probes. Some ABPs, such as UbPrg and Ub-VME, can also label active Cys in HECT E3 ligases (Ekkebus et al., 2013).

Recently published cascading E1-E2-E3 ABP (Mulder et al., 2016) is based on the use of the Ub variant in which Gly76 is replaced by dehydroalanine (Dha) that can be processed by the cellular ubiquitination machinery (Hodgins et al., 1992; Pickart et al., 1994; Mulder et al., 2016). Once added to lysate or electroporated into cells, UbDha is activated by E1 enzyme (through the formation of an adenylate intermediate) and the activated reactive methylene group of the Dha moiety can then either covalently trap the enzyme in an E1-UbDha thioether adduct or follow the native resulting in an $\mathrm{E} 1 \sim \mathrm{UbDh}$ thioester. Such thioester can then be transferred to an E2 enzyme and either form covalent thioether adduct with the probe or undergo native trans-thioesterification. Following the ubiquitination pathway UbDha can subsequently be transferred to an active site of either HECT or RBR E3 ligases.

In this manner, the probe can travel through the entire E1E2-E3 cascade, where it "traps" catalytically active Ub-modifying enzymes along the way. Unlike endogenous $\mathrm{Ub}$, the probe can irreversibly react with the active site Cys residue of target enzymes in living cells. It can be also combined with MS to identify or quantify E1-E2-E3 cellular activities. However, in its current form the probe is not selective for specific E2 or E3 enzymes and cannot capture RING E3 ligases in a mechanism-dependent manner (Mulder et al., 2016), which requires novel probe designs.

Pao et al. (2018) have recently developed a novel ABP consisting of Ub-charged E2 conjugate with C-terminal activated

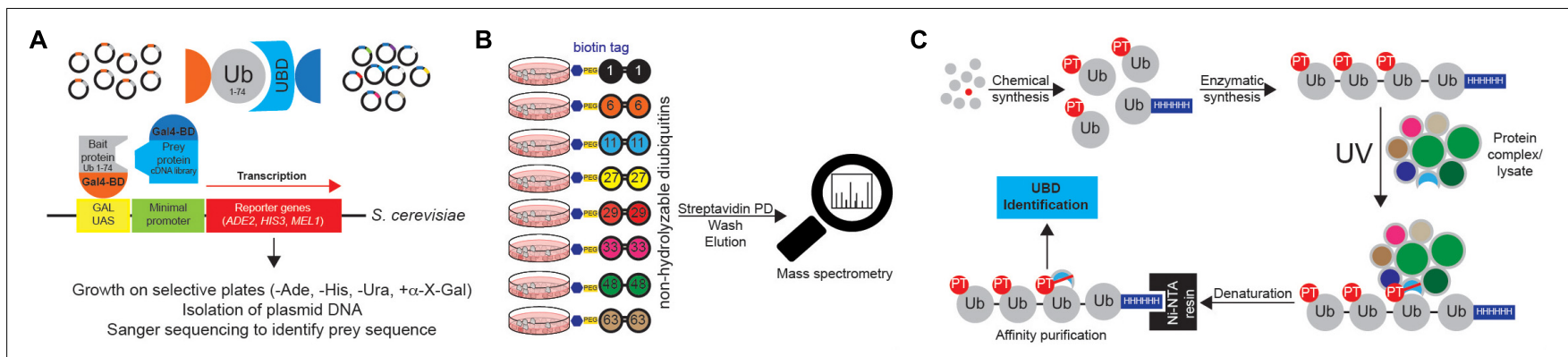

FIGURE 10 | Approaches to identify ubiquitin receptors. (A) A very common type of $\mathrm{Y} 2 \mathrm{H}$ approach is based on the use of Ub sequence (lacking C-terminal Gly-Gly motif) as N-terminal fusion with the GAL4 binding domain (BD). The cDNA library containing putative UBDs consists of cDNAs cloned under the control of the lacZ promoter downstream of the DNA sequence encoding the activating domain (AD) of the yeast GAL4 transcription factor. Protein interaction induces the close proximity of GAL4AD and GAL4BD, forming the active transcription factor, which binds to the GAL1 upstream activating sequence (UAS) and activates the transcription of several GAL4-responsive genes, which are used as reporters. For example, yeast strain S. cerevisiae YTHGold (Clontech) enables very stringent quadruple selection, since it contains 4 reporters. In that way background growth and detection of false positive interacting proteins is significantly decreased, simplifying further evaluation steps. (B) UbIA-MS method relies on the use of 8 chemically synthesized non-hydrolyzable biotinylated diUbs that can be used for in vitro affinity purification of Ub interactors. These diUb linkages mimic native diUb, and have advantage of not being cleaved by cellular DUBs, which prevents the loss of captured material and decrease in Ub chain specificity. Upon purification, samples are digested on beads with trypsin, followed by liquid chromatography (LC)-MS/MS analysis. (C) Ub-PT is a synthetic Ub variant that contains a photo-activatable crosslinking Leu mimic photoleucine (pLeu) at positions 8 or 73 in Ub molecule. Importantly, these modifications do not affect Ub functionality, including its ability to bind UBDs. Enzymatic polymerization of Ub-PT into Ub chains of defined lengths and linkage types allows the use of Ub-PT as UV-activatable crosslinking reagent (phototrap) for irreversibly capturing Ub receptors. Furthermore, the existence of 6xHIS tag in Ub-PT-containing reagents allows stringent isolation of Ub interactors, without co-purification of their binding proteins. 
vinylsulfide (E2-Ub-AVS) as the warhead for the detection and identification of novel E3 ligases. By using their probe, they identified MYCBP2/PHR1 as so far unique E3 ligase with esterification activity and intrinsic selectivity for Thr over Ser (Pao et al., 2018). Like E1-E2-E3 probe, this approach also lacks the ability to study specific E3 ligases and to monitor RING E3 ligase family.

Additionally, Dha-based E2-Ub ABP was also developed for monitoring HECT E3 activity in vitro and in vivo (Xu et al., 2019).

The UPS-confocal fluorescence nanoscanning (UPSCONA) assay is based on the immobilization of the specific substrate of interest on micro-beads. Fluorescently labeled $\mathrm{Ub}$ is enzymatically conjugated to the substrate and can be quantitatively detected on the bead periphery by confocal microscopy. UPS-CONA approach can be used for studying specific enzymes of the ubiquitination machinery, as well as for measuring the selectivity of putative ubiquitination inhibitors (Koszela et al., 2018).

\section{Methods to Study Non-covalent Ubiquitin Recognition Identification of Novel Ubiquitin Readers and Determination of Their Specificity Toward Ubiquitin}

The number of currently known Ub readers is relatively low in comparison to the number of proteins playing active roles in ubiquitination and deubiquitination or those modified by various $\mathrm{Ub}$ moieties. It is reasonable to speculate that there are still multiple UBDs that remain unknown at the moment, especially those specific for Ub linkages, those that are Ub chain lengthdependent or can specifically recognize heterotypic/branched Ub linkages or even specific Ub PTMs.

The use of single Ub moiety lacking diGly motif at the $\mathrm{C}$ terminus (to prevent potential conjugation to yeast proteins) as bait in yeast two-hybrid ( $\mathrm{Y} 2 \mathrm{H})$ screen led to the identification of several novel UBD-containing proteins, including Pru domain of RPN13 (Figure 10A) (Bienko et al., 2005; Husnjak et al., 2008; Wagner et al., 2008). This approach is limited to UBDs that bind single Ub moieties and cannot be used for Ub linkagespecific UBDs.

Ub chains cannot be efficiently used for the AP of Ub chainspecific UBDs, as cellular DUBs readily cleave them. However, by combining chemically synthesized non-hydrolyzable diUb molecules with AP/MS, Zhang et al. (2017) could successfully enrich and identify many known and novel Ub interactors with simultaneous evaluation of their Ub linkage specificity (Figure 10B). The novel method, termed Ub interactor affinity enrichment-MS (UbIA-MS) identified TAB2 and TAB3 as novel Lys6 interactors and characterized UCHL3 as Lys27-specific Ub receptor (Zhang et al., 2017).

Another promising approach for identifying novel UBDs is the use of synthetic Ub variant that contains a photoactivatable crosslinking side chain. Photoleucine (pLeu) incorporated into fully synthetic Ub monomer does not prevent UBD binding to its Ile44 patch and can be readily incorporated into polyUb chain, without affecting the specificity of the binding (Figure 10C). Once photoactivated by UV, it is able to crosslink nearby proteins, thus stabilizing often very weak UBD:Ub interactions. By using Ub-PT as a tool, Chojnacki et al. (2017) identified proteasomal subunit Rpn1 as a novel Ub receptor. Moreover, Ub-PT can have pLeu incorporated at multiple positions within Ub molecule, making it very useful for capturing UBDs that interact in different ways with $\mathrm{Ub}$.

\section{CONCLUDING REMARKS}

Ub field has been extensively studied in the last several decades, and its recognition as the promising drug target has initiated a large number of studies aiming to improve our understanding of the complex nature of ubiquitination regulation. Novel DUB inhibitors have been developed, as well as multiple tools to identify novel components (enzymes, scaffolds, and receptors), to study enzymatic activity, cellular distribution, modes of regulation and potential chemical inhibition of Ub system.

The use of proteolysis targeting chimeras (PROTACs) for induction of specific protein degradation has emerged as promising approach for targeting proteins that are otherwise hard or impossible to target by small molecule approaches (Watt et al., 2019), making further research of the Ub system a priority. Still, there are many things that we do not know or that we have just started to elucidate. Newly developed tools and approaches will clearly shed a new light on our understanding of Ub systems and ways how we can explore it for treating diseases.

\section{OUTSTANDING QUESTIONS}

There are still no straightforward approaches for determining E3 ligases responsible for specific ubiquitination events. Furthermore, ABPs specific for single E3 ligases (and many DUBs) are still missing, as well as small molecules that can either specifically inhibit (or potentially activate) these enzymes.

Even though numerous high-throughput MS studies have identified tens of thousands of ubiquitination sites at the proteome level (Kim et al., 2011; Wagner et al., 2011), for only a small proportion of these modifications a specific E3 ligase is known. Methods such as Ub remnant profiling do not allow identification of E3 ligases that modify specific Lys residues on identified proteins.

Due to transient interaction between E3 ligases and their substrates, standard approaches (such as immunoprecipitation) are not appropriate for identification of specific E3 ligases:substrate pairs, even more so since many (but not all) E3 ligases target their substrates for proteasomal degradation, thus reducing their levels. In line with that, protein abundance upon E3 ligase removal/inhibition or activation/overexpression cannot be a good readout for identifying specific E3 ligase substrates.

As many E3 ligases are multicomponent complexes, whose activation often depends on various PTMs, many of these enzymes are difficult to be used in various high-throughput screening efforts for their regulators. As ubiquitination is a very complex PTM, with numerous heterotypic/branched and combinatorial ubiquitination events, it is clear that all the available methodology is still unable to fully comprehend the extent and importance of these modifications. 


\section{AUTHOR CONTRIBUTIONS}

Both authors listed have made a substantial, direct and intellectual contribution to the work, and approved it for publication.

\section{REFERENCES}

Akimov, V., Barrio-Hernandez, I., Hansen, S. V. F., Hallenborg, P., Pedersen, A. K., Bekker-Jensen, D. B., et al. (2018a). UbiSite approach for comprehensive mapping of lysine and N-terminal ubiquitination sites. Nat. Struct. Mol. Biol. 25, 631-640. doi: 10.1038/s41594-018-0084-y

Akimov, V., Olsen, L. C. B., Hansen, S. V. F., Barrio-Hernandez, I., Puglia, M., Jensen, S. S., et al. (2018b). StUbEx PLUS-A modified stable tagged ubiquitin exchange system for peptide level purification and in-depth mapping of ubiquitination sites. J. Proteome Res. 17, 296-304. doi: 10.1021/acs.jproteome. $7 \mathrm{~b} 00566$

Akutsu, M., Dikic, I., and Bremm, A. (2016). Ubiquitin chain diversity at a glance. J. Cell Sci. 129, 875-880. doi: 10.1242/jcs.183954

Beaudette, P., Popp, O., and Dittmar, G. (2016). Proteomic techniques to probe the ubiquitin landscape. Proteomics 16, 273-287. doi: 10.1002/pmic.201500290

Bekes, M., Okamoto, K., Crist, S. B., Jones, M. J., Chapman, J. R., Brasher, B. B., et al. (2013). DUB-resistant ubiquitin to survey ubiquitination switches in mammalian cells. Cell Rep. 5, 826-838. doi: 10.1016/j.celrep.2013.10.008

Bennett, E. J., Shaler, T. A., Woodman, B., Ryu, K. Y., Zaitseva, T. S., Becker, C. H., et al. (2007). Global changes to the ubiquitin system in Huntington's disease. Nature 448, 704-708.

Bhogaraju, S., Bonn, F., Mukherjee, R., Adams, M., Pfleiderer, M. M., Galej, W. P., et al. (2019). Inhibition of bacterial ubiquitin ligases by SidJ-calmodulin catalysed glutamylation. Nature 572, 382-386. doi: 10.1038/s41586-019-1440-8

Bhogaraju, S., Kalayil, S., Liu, Y., Bonn, F., Colby, T., Matic, I., et al. (2016). Phosphoribosylation of ubiquitin promotes serine ubiquitination and impairs conventional ubiquitination. Cell 167, 1636-1649.e13. doi: 10.1016/j.cell.2016. 11.019

Bienko, M., Green, C. M., Crosetto, N., Rudolf, F., Zapart, G., Coull, B., et al. (2005). Ubiquitin-binding domains in Y-family polymerases regulate translesion synthesis. Science 310, 1821-1824.

Bonacci, T., Suzuki, A., Grant, G. D., Stanley, N., Cook, J. G., Brown, N. G., et al. (2018). Cezanne/OTUD7B is a cell cycle-regulated deubiquitinase that antagonizes the degradation of APC/C substrates. EMBO J. 37:e98701. doi: 10.15252/embj.201798701

Borodovsky, A., Kessler, B. M., Casagrande, R., Overkleeft, H. S., Wilkinson, K. D., and Ploegh, H. L. (2001). A novel active site-directed probe specific for deubiquitylating enzymes reveals proteasome association of USP14. EMBO J. 20, 5187-5196.

Borodovsky, A., Ovaa, H., Kolli, N., Gan-Erdene, T., Wilkinson, K. D., Ploegh, H. L., et al. (2002). Chemistry-based functional proteomics reveals novel members of the deubiquitinating enzyme. Chem. Biol. 9, 1149-1159.

Braten, O., Livneh, I., Ziv, T., Admon, A., Kehat, I., Caspi, L. H., et al. (2016). Numerous proteins with unique characteristics are degraded by the $26 \mathrm{~S}$ proteasome following monoubiquitination. Proc. Natl. Acad. Sci. U.S.A. 113, E4639-E4647. doi: 10.1073/pnas.1608644113

Cadwell, K., and Coscoy, L. (2005). Ubiquitination on nonlysine residues by a viral E3 ubiquitin ligase. Science 309, 127-130.

Chaugule, V. K., Burchell, L., Barber, K. R., Sidhu, A., Leslie, S. J., Shaw, G. S., et al. (2011). Autoregulation of parkin activity through its ubiquitin-like domain. EMBO J. 30, 2853-2867. doi: 10.1038/emboj.2011.204

Chen, X., Lu, D., Gao, J., Zhu, H., Zhou, Y., Gao, D., et al. (2019). Identification of a USP9X substrate NFX1-123 by SILAC-Based quantitative proteomics. J. Proteome. Res. 18, 2654-2665. doi: 10.1021/acs.jproteome.9b00139

Chen, B. B., and Mallampalli, R. K. (2009). Masking of a nuclear signal motif by monoubiquitination leads to mislocalization and degradation of the regulatory enzyme cytidylyltransferase. Mol. Cell. Biol. 29, 3062-3075. doi: 10.1128/MCB. 01824-08

Chen, Z., Zhong, Y., Wang, Y., Xu, S., Liu, Z., Baskakov, I. V., et al. (2013). Ubiquitination-induced fluorescence complementation (UiFC) for detection of K48 ubiquitin chains in vitro and in live cells. PLoS One 8:e73482. doi: 10.1371/journal.pone.0073482

\section{ACKNOWLEDGMENTS}

We apologize to all the authors whose valuable work could not have been mentioned in this article due to size limitations.

Chojnacki, M., Mansour, W., Hameed, D. S., Singh, R. K., El Oualid, F., Rosenzweig, R., et al. (2017). Polyubiquitin-photoactivatable crosslinking reagents for mapping ubiquitin interactome identify Rpn1 as a proteasome ubiquitin-associating subunit. Cell Chem. Biol. 24, 443-457.e6. doi: 10.1016/j. chembiol.2017.02.013

Ciechanover, A., and Ben-Saadon, R. (2004). N-terminal ubiquitination: more protein substrates join in. Trends Cell Biol. 14, 103-106.

Coyaud, E., Mis, M., Laurent, E. M., Dunham, W. H., Couzens, A. L., Robitaille, M., et al. (2015). BioID-based identification of Skp cullin F-box (SCF)beta-TrCP1/2 E3 ligase substrates. Mol. Cell. Proteomics 14, 1781-1795. doi: 10.1074/mcp. M114.045658

Cromm, P. M., and Crews, C. M. (2017). Targeted protein degradation: from chemical biology to drug discovery. Cell Chem. Biol. 24, 1181-1190. doi: 10 . 1016/j.chembiol.2017.05.024

Crowe, S. O., Rana, A., Deol, K. K., Ge, Y., and Strieter, E. R. (2017). Ubiquitin chain enrichment middle-down mass spectrometry enables characterization of branched ubiquitin chains in cellulo. Anal. Chem. 89, 4428-4434. doi: 10.1021/ acs.analchem.6b03675

Cui, J., Yao, Q., Li, S., Ding, X., Lu, Q., Mao, H., et al. (2010). Glutamine deamidation and dysfunction of ubiquitin/NEDD8 induced by a bacterial effector family. Science 329, 1215-1218. doi: 10.1126/science.1193844

Danielsen, J. M., Sylvestersen, K. B., Bekker-Jensen, S., Szklarczyk, D., Poulsen, J. W., Horn, H., et al. (2011). Mass spectrometric analysis of lysine ubiquitylation reveals promiscuity at site level. Mol. Cell. Proteomics 10:M110003590. doi: 10.1074/mcp.M110.003590

Danielson, C. M., and Hope, T. J. (2013). Using antiubiquitin antibodies to probe the ubiquitination state within rhTRIM5alpha cytoplasmic bodies. AIDS Res. Hum. Retroviruses 29, 1373-1385. doi: 10.1089/AID.2013. 0029

de Jong, A., Merkx, R., Berlin, I., Rodenko, B., Wijdeven, R. H., El Atmioui, D., et al. (2012). Ubiquitin-based probes prepared by total synthesis to profile the activity of deubiquitinating enzymes. Chembiochem 13, 2251-2258. doi: 10.1002/cbic. 201200497

Ekkebus, R., van Kasteren, S. I., Kulathu, Y., Scholten, A., Berlin, I., Geurink, P. P., et al. (2013). On terminal alkynes that can react with active-site cysteine nucleophiles in proteases. J. Am. Chem. Soc. 135, 2867-2870. doi: 10.1021/ ja309802n

El Oualid, F., Merkx, R., Ekkebus, R., Hameed, D. S., Smit, J. J., de Jong, A., et al. (2010). Chemical synthesis of ubiquitin, ubiquitin-based probes, and diubiquitin. Angew. Chem. 49, 10149-10153.

Emmerich, C. H., Bakshi, S., Kelsall, I. R., Ortiz-Guerrero, J., Shpiro, N., and Cohen, P. (2016). Lys63/Met1-hybrid ubiquitin chains are commonly formed during the activation of innate immune signalling. Biochem. Biophys. Res. Commun. 474, 452-461. doi: 10.1016/j.bbrc.2016.04.141

Emmerich, C. H., and Cohen, P. (2015). Optimising methods for the preservation, capture and identification of ubiquitin chains and ubiquitylated proteins by immunoblotting. Biochem. Biophys. Res. Commun. 466, 1-14. doi: 10.1016/j. bbrc.2015.08.109

Emmerich, C. H., Ordureau, A., Strickson, S., Arthur, J. S., Pedrioli, P. G., Komander, D., et al. (2013). Activation of the canonical IKK complex by K63/M1-linked hybrid ubiquitin chains. Proc. Natl. Acad. Sci. U.S.A. 110, 15247-15252. doi: 10.1073/pnas.1314715110

Fiil, B. K., Damgaard, R. B., Wagner, S. A., Keusekotten, K., Fritsch, M., BekkerJensen, S., et al. (2013). OTULIN restricts Met1-linked ubiquitination to control innate immune signaling. Mol. Cell 50, 818-830. doi: 10.1016/j.molcel.2013.06. 004

Finley, D., Bartel, B., and Varshavsky, A. (1989). The tails of ubiquitin precursors are ribosomal proteins whose fusion to ubiquitin facilitates ribosome biogenesis. Nature 338, 394-401.

Fiskin, E., Bionda, T., Dikic, I., and Behrends, C. (2016). Global analysis of host and bacterial ubiquitinome in response to Salmonella typhimurium infection. Mol. Cell 62, 967-981. doi: 10.1016/j.molcel.2016.04.015 
Flierman, D., van der Heden van Noort, G. J., Ekkebus, R., Geurink, P. P., Mevissen, T. E., Hospenthal, M. K., et al. (2016). Non-hydrolyzable diubiquitin probes reveal linkage-specific reactivity of deubiquitylating enzymes mediated by S2 pockets. Cell Chem. Biol. 23, 472-482. doi: 10.1016/j.chembiol.2016.03.009

Franco, M., Seyfried, N. T., Brand, A. H., Peng, J., and Mayor, U. (2011). A novel strategy to isolate ubiquitin conjugates reveals wide role for ubiquitination during neural development. Mol. Cell. Proteomics 10:M110002188. doi: 10. 1074/mcp.M110.002188

Fujimuro, M., Sawada, H., and Yokosawa, H. (1994). Production and characterization of monoclonal antibodies specific to multi-ubiquitin chains of polyubiquitinated proteins. FEBS Lett. 349, 173-180.

Galisson, F., Mahrouche, L., Courcelles, M., Bonneil, E., Meloche, S., Chelbi-Alix, M. K., et al. (2011). A novel proteomics approach to identify SUMOylated proteins and their modification sites in human cells. Mol. Cell. Proteomics 10:M110004796. doi: 10.1074/mcp.M110.004796

Gao, Y., Li, Y., Zhang, C., Zhao, M., Deng, C., Lan, Q., et al. (2016). Enhanced purification of ubiquitinated proteins by engineered tandem hybrid ubiquitinbinding domains (ThUBDs). Mol. Cell. Proteomics 15, 1381-1396. doi: 10.1074/ mcp.O115.051839

Geurink, P. P., van Tol, B. D., van Dalen, D., Brundel, P. J., Mevissen, T. E., Pruneda, J. N., et al. (2016). Development of diubiquitin-based FRET probes to quantify ubiquitin linkage specificity of deubiquitinating enzymes. Chembiochem 17, 816-820. doi: 10.1002/cbic.201600017

Grice, G. L., Lobb, I. T., Weekes, M. P., Gygi, S. P., Antrobus, R., and Nathan, J. A. (2015). The proteasome distinguishes between heterotypic and homotypic lysine-11-linked polyubiquitin chains. Cell Rep. 12, 545-553. doi: 10.1016/j. celrep.2015.06.061

Grou, C. P., Pinto, M. P., Mendes, A. V., Domingues, P., and Azevedo, J. E. (2015). The de novo synthesis of ubiquitin: identification of deubiquitinases acting on ubiquitin precursors. Sci. Rep. 5:12836. doi: 10.1038/srep 12836

Harrigan, J. A., Jacq, X., Martin, N. M., and Jackson, S. P. (2018). Deubiquitylating enzymes and drug discovery: emerging opportunities. Nat. Rev. Drug Discov. 17, 57-78. doi: 10.1038/nrd.2017.152

Hendriks, I. A., D’Souza, R. C., Yang, B., Verlaan-de Vries, M., Mann, M., and Vertegaal, A. C. (2014). Uncovering global SUMOylation signaling networks in a site-specific manner. Nat. Struct. Mol. Biol. 21, 927-936. doi: 10.1038/nsmb. 2890

Herhaus, L., and Dikic, I. (2015). Expanding the ubiquitin code through posttranslational modification. EMBO Rep. 16, 1071-1083. doi: 10.15252/embr. 201540891

Hershko, A., and Ciechanover, A. (1998). The ubiquitin system. Annu. Rev. Biochem. 67, 425-479.

Hjerpe, R., Aillet, F., Lopitz-Otsoa, F., Lang, V., England, P., and Rodriguez, M. S. (2009). Efficient protection and isolation of ubiquitylated proteins using tandem ubiquitin-binding entities. EMBO Rep. 10, 1250-1258. doi: 10.1038/ embor.2009.192

Hodgins, R. R., Ellison, K. S., and Ellison, M. J. (1992). Expression of a ubiquitin derivative that conjugates to protein irreversibly produces phenotypes consistent with a ubiquitin deficiency. J. Biol. Chem. 267, 8807-8812.

Hospenthal, M. K., Mevissen, T. E., and Komander, D. (2015). Deubiquitinasebased analysis of ubiquitin chain architecture using Ubiquitin Chain Restriction (UbiCRest). Nat. Protoc. 10, 349-361. doi: 10.1038/nprot.2015.018

Husnjak, K., and Dikic, I. (2012). Ubiquitin-binding proteins: decoders of ubiquitin-mediated cellular functions. Annu. Rev. Biochem. 81, 291-322. doi: 10.1146/annurev-biochem-051810-094654

Husnjak, K., Elsasser, S., Zhang, N., Chen, X., Randles, L., Shi, Y., et al. (2008). Proteasome subunit Rpn13 is a novel ubiquitin receptor. Nature 453, 481-488. doi: 10.1038 /nature 06926

Iconomou, M., and Saunders, D. N. (2016). Systematic approaches to identify E3 ligase substrates. Biochem. J. 473, 4083-4101.

Ikeda, F., Deribe, Y. L., Skanland, S. S., Stieglitz, B., Grabbe, C., Franz-Wachtel, M., et al. (2011). SHARPIN forms a linear ubiquitin ligase complex regulating NF-kappaB activity and apoptosis. Nature 471, 637-641. doi: 10.1038/nature 09814

Johnson, A., and Vert, G. (2016). Unraveling K63 polyubiquitination networks by sensor-based proteomics. Plant Physiol. 171, 1808-1820. doi: 10.1104/pp.16. 00619
Kaiser, S. E., Riley, B. E., Shaler, T. A., Trevino, R. S., Becker, C. H., Schulman, H., et al. (2011). Protein standard absolute quantification (PSAQ) method for the measurement of cellular ubiquitin pools. Nat. Methods 8, 691-696. doi: 10.1038/nmeth.1649

Kane, L. A., Lazarou, M., Fogel, A. I., Li, Y., Yamano, K., Sarraf, S. A., et al. (2014). PINK1 phosphorylates ubiquitin to activate Parkin E3 ubiquitin ligase activity. J. Cell Biol. 205, 143-153. doi: 10.1083/jcb.201402104

Kazlauskaite, A., Kondapalli, C., Gourlay, R., Campbell, D. G., Ritorto, M. S., Hofmann, K., et al. (2014). Parkin is activated by PINK1-dependent phosphorylation of ubiquitin at Ser ${ }^{65}$. Biochem. J. 460, 127-139. doi: 10.1042/ BJ20140334

Kim, H. C., and Huibregtse, J. M. (2009). Polyubiquitination by HECT E3s and the determinants of chain type specificity. Mol. Cell. Biol. 29, 3307-3318. doi: 10.1128/MCB.00240-09

Kim, W., Bennett, E. J., Huttlin, E. L., Guo, A., Li, J., Possemato, A., et al. (2011). Systematic and quantitative assessment of the ubiquitin-modified proteome. Mol. Cell 44, 325-340. doi: 10.1016/j.molcel.2011.08.025

Kirisako, T., Kamei, K., Murata, S., Kato, M., Fukumoto, H., Kanie, M., et al. (2006). A ubiquitin ligase complex assembles linear polyubiquitin chains. EMBO J. 25, 4877-4887.

Kirkpatrick, D. S., Gerber, S. A., and Gygi, S. P. (2005). The absolute quantification strategy: a general procedure for the quantification of proteins and posttranslational modifications. Methods 35, 265-273.

Kliza, K., Taumer, C., Pinzuti, I., Franz-Wachtel, M., Kunzelmann, S., Stieglitz, B., et al. (2017). Internally tagged ubiquitin: a tool to identify linear polyubiquitinmodified proteins by mass spectrometry. Nat. Methods 14, 504-512. doi: 10. 1038/nmeth. 4228

Komander, D., Clague, M. J., and Urbe, S. (2009). Breaking the chains: structure and function of the deubiquitinases. Nat. Rev. Mol. Cell Biol. 10, 550-563. doi: $10.1038 / \mathrm{nrm} 2731$

Kondapalli, C., Kazlauskaite, A., Zhang, N., Woodroof, H. I., Campbell, D. G., Gourlay, R., et al. (2012). PINK1 is activated by mitochondrial membrane potential depolarization and stimulates Parkin E3 ligase activity by phosphorylating Serine 65. Open Biol. 2:120080. doi: 10.1098/rsob.120080

Koszela, J., Pham, N. T., Evans, D., Mann, S., Perez-Pi, I., Shave, S., et al. (2018). Real-time tracking of complex ubiquitination cascades using a fluorescent confocal on-bead assay. BMC Biol. 16:88. doi: 10.1186/s12915-018-0554-z

Kotewicz, K. M., Ramabhadran, V., Sjoblom, N., Vogel, J. P., Haenssler, E., Zhang, M., et al. (2017). A single legionella effector catalyzes a multistep ubiquitination pathway to rearrange tubular endoplasmic reticulum for replication. Cell Host Microbe 21, 169-181. doi: 10.1016/j.chom.2016.12.007

Koyano, F., Okatsu, K., Kosako, H., Tamura, Y., Go, E., Kimura, M., et al. (2014). Ubiquitin is phosphorylated by PINK1 to activate parkin. Nature 510, 162-166. doi: 10.1038/nature13392

Kulathu, Y., and Komander, D. (2012). Atypical ubiquitylation - the unexplored world of polyubiquitin beyond Lys48 and Lys63 linkages. Nat. Rev. Mol. Cell Biol. 13, 508-523. doi: 10.1038/nrm3394

Lafont, E., Kantari-Mimoun, C., Draber, P., De Miguel, D., Hartwig, T., Reichert, M., et al. (2017). The linear ubiquitin chain assembly complex regulates TRAILinduced gene activation and cell death. EMBO J. 36, 1147-1166. doi: 10.15252/ embj. 201695699

Lamoliatte, F., Bonneil, E., Durette, C., Caron-Lizotte, O., Wildemann, D., Zerweck, J., et al. (2013). Targeted identification of SUMOylation sites in human proteins using affinity enrichment and paralog-specific reporter ions. Mol. Cell. Proteomics 12, 2536-2550. doi: 10.1074/mcp.M112.025569

Lectez, B., Migotti, R., Lee, S. Y., Ramirez, J., Beraza, N., Mansfield, B., et al. (2014). Ubiquitin profiling in liver using a transgenic mouse with biotinylated ubiquitin. J. Proteome Res. 13, 3016-3026. doi: 10.1021/pr5001913

Li, G., Liang, Q., Gong, P., Tencer, A. H., and Zhuang, Z. (2014). Activity-based diubiquitin probes for elucidating the linkage specificity of deubiquitinating enzymes. Chem. Commun. 50, 216-218. doi: 10.1039/c3cc47382a

Lim, M., Newman, J. A., Williams, H. L., Masino, L., Aitkenhead, H., Gravard, A. E., et al. (2019). A ubiquitin-binding domain that binds a structural fold distinct from that of ubiquitin. Structure 27, 1316-1325.e6. doi: 10.1016/j.str.2019.05. 003

Liu, Z., Gong, Z., Jiang, W. X., Yang, J., Zhu, W. K., Guo, D. C., et al. (2015). Lys63linked ubiquitin chain adopts multiple conformational states for specific target recognition. eLife 4:e05767. 
Loveless, T. B., Topacio, B. R., Vashisht, A. A., Galaang, S., Ulrich, K. M., Young, B. D., et al. (2015). DNA damage regulates translation through betaTRCP targeting of CReP. PLoS Genet. 11:e1005292. doi: 10.1371/journal.pgen. 1005292

Maculins, T., Fiskin, E., Bhogaraju, S., and Dikic, I. (2016). Bacteria-host relationship: ubiquitin ligases as weapons of invasion. Cell Res. 26, 499-510. doi: $10.1038 /$ cr.2016.30

Marblestone, J. G., Larocque, J. P., Mattern, M. R., and Leach, C. A. (2012). Analysis of ubiquitin E3 ligase activity using selective polyubiquitin binding proteins. Biochim. Biophys. Acta 1823, 2094-2097. doi: 10.1016/j.bbamcr.2012.06.013

Mark, K. G., Loveless, T. B., and Toczyski, D. P. (2016). Isolation of ubiquitinated substrates by tandem affinity purification of E3 ligase-polyubiquitin-binding domain fusions (ligase traps). Nat. Protoc. 11, 291-301. doi: 10.1038/nprot.2016. 008

Mark, K. G., Simonetta, M., Maiolica, A., Seller, C. A., and Toczyski, D. P. (2014). Ubiquitin ligase trapping identifies an $\operatorname{SCF}($ Saf1) pathway targeting unprocessed vacuolar/lysosomal proteins. Mol. Cell 53, 148-161. doi: 10.1016/j. molcel.2013.12.003

Matsumoto, G., Wada, K., Okuno, M., Kurosawa, M., and Nukina, N. (2011). Serine 403 phosphorylation of p62/SQSTM1 regulates selective autophagic clearance of ubiquitinated proteins. Mol. Cell 44, 279-289. doi: 10.1016/j. molcel.2011.07.039

Matsumoto, M., Hatakeyama, S., Oyamada, K., Oda, Y., Nishimura, T., and Nakayama, K. I. (2005). Large-scale analysis of the human ubiquitin-related proteome. Proteomics 5, 4145-4151.

Matsumoto, M. L., Dong, K. C., Yu, C., Phu, L., Gao, X., Hannoush, R. N., et al. (2012). Engineering and structural characterization of a linear polyubiquitinspecific antibody. J. Mol. Biol. 418, 134-144. doi: 10.1016/j.jmb.2011.12.053

Matsumoto, M. L., Wickliffe, K. E., Dong, K. C., Yu, C., Bosanac, I., Bustos, D., et al. (2010). K11-linked polyubiquitination in cell cycle control revealed by a K11 linkage-specific antibody. Mol. Cell 39, 477-484. doi: 10.1016/j.molcel.2010.07. 001

Mattern, M., Sutherland, J., Kadimisetty, K., Barrio, R., and Rodriguez, M. S. (2019). Using ubiquitin binders to decipher the ubiquitin code. Trends Biochem. Sci. 44, 599-615. doi: 10.1016/j.tibs.2019.01.011

McDowell, G. S., and Philpott, A. (2016). New insights into the role of ubiquitylation of proteins. Int. Rev. Cell Mol. Biol. 325, 35-88. doi: 10.1016/bs. ircmb.2016.02.002

McGouran, J. F., Gaertner, S. R., Altun, M., Kramer, H. B., and Kessler, B. M. (2013). Deubiquitinating enzyme specificity for ubiquitin chain topology profiled by diubiquitin activity probes. Chem. Biol. 20, 1447-1455. doi: 10.1016/j.chembiol. 2013.10.012

Meierhofer, D., Wang, X., Huang, L., and Kaiser, P. (2008). Quantitative analysis of global ubiquitination in HeLa cells by mass spectrometry. J. Proteome Res. 7, 4566-4576. doi: 10.1021/pr800468j

Metzger, M. B., Hristova, V. A., and Weissman, A. M. (2012). HECT and RING finger families of E3 ubiquitin ligases at a glance. J. Cell Sci. 125, 531-537.

Mevissen, T. E. T., and Komander, D. (2017). Mechanisms of deubiquitinase specificity and regulation. Annu. Rev. Biochem. 86, 159-192. doi: 10.1146/ annurev-biochem-061516-044916

Meyer, H. J., and Rape, M. (2014). Enhanced protein degradation by branched ubiquitin chains. Cell 157, 910-921. doi: 10.1016/j.cell.2014.03.037

Michel, M. A., Swatek, K. N., Hospenthal, M. K., and Komander, D. (2017). Ubiquitin linkage-specific affimers reveal insights into K6-linked ubiquitin signaling. Mol. Cell 68, 233-246.e5. doi: 10.1016/j.molcel.2017.08.020

Mulder, M. P., El Oualid, F., ter Beek, J., and Ovaa, H. (2014). A native chemical ligation handle that enables the synthesis of advanced activity-based probes: diubiquitin as a case study. Chembiochem 15, 946-949. doi: 10.1002/cbic. 201402012

Mulder, M. P., Witting, K., Berlin, I., Pruneda, J. N., Wu, K. P., Chang, J. G., et al. (2016). A cascading activity-based probe sequentially targets E1-E2-E3 ubiquitin enzymes. Nat. Chem. Biol. 12, 523-530. doi: 10.1038/nchembio.2084

Nakayasu, E. S., Sydor, M. A., Brown, R. N., Sontag, R. L., Sobreira, T. J., Slysz, G. W., et al. (2015). Identification of Salmonella typhimurium deubiquitinase SseL substrates by immunoaffinity enrichment and quantitative proteomic analysis. J. Proteome Res. 14, 4029-4038. doi: 10.1021/acs.jproteome.5b00574

Nakazawa, S., Oikawa, D., Ishii, R., Ayaki, T., Takahashi, H., Takeda, H., et al. (2016). Linear ubiquitination is involved in the pathogenesis of optineurinassociated amyotrophic lateral sclerosis. Nat. Commu. 7:12547. doi: 10.1038/ ncomms 12547
Newton, K., Matsumoto, M. L., Ferrando, R. E., Wickliffe, K. E., Rape, M., Kelley, R. F., et al. (2012). Using linkage-specific monoclonal antibodies to analyze cellular ubiquitylation. Methods Mol. Biol. 832, 185-196. doi: 10.1007/978-161779-474-2_13

Newton, K., Matsumoto, M. L., Wertz, I. E., Kirkpatrick, D. S., Lill, J. R., Tan, J., et al. (2008). Ubiquitin chain editing revealed by polyubiquitin linkage-specific antibodies. Cell 134, 668-678. doi: 10.1016/j.cell.2008.07.039

Nielsen, M. L., Vermeulen, M., Bonaldi, T., Cox, J., Moroder, L., and Mann, M. (2008). Iodoacetamide-induced artifact mimics ubiquitination in mass spectrometry. Nat. Methods 5, 459-460.

O’Connor, H. F., Lyon, N., Leung, J. W., Agarwal, P., Swaim, C. D., Miller, K. M., et al. (2015). Ubiquitin-activated interaction traps (UBAITs) identify E3 ligase binding partners. EMBO Rep. 16, 1699-1712. doi: 10.15252/embr.201540620

O'Connor, H. F., Swaim, C. D., Canadeo, L. A., and Huibregtse, J. M. (2018). Ubiquitin-activated interaction traps (UBAITs): tools for capturing proteinprotein interactions. Methods Mol. Biol. 1844, 85-100. doi: 10.1007/978-14939-8706-1_7

Ohtake, F., Saeki, Y., Sakamoto, K., Ohtake, K., Nishikawa, H., Tsuchiya, H., et al. (2015). Ubiquitin acetylation inhibits polyubiquitin chain elongation. $E M B O$ Rep. 16, 192-201. doi: 10.15252/embr.201439152

Okatsu, K., Sato, Y., Yamano, K., Matsuda, N., Negishi, L., Takahashi, A., et al. (2018). Structural insights into ubiquitin phosphorylation by PINK1. Sci. Rep. 8:10382. doi: 10.1038/s41598-018-28656-8

Ordureau, A., Paulo, J. A., Zhang, W., Ahfeldt, T., Zhang, J., Cohn, E. F., et al. (2018). Dynamics of PARKIN-dependent mitochondrial ubiquitylation in induced neurons and model systems revealed by digital snapshot proteomics. Mol. Cell 70, 211-227.e8. doi: 10.1016/j.molcel.2018.03.012

Ozkaynak, E., Finley, D., and Varshavsky, A. (1984). The yeast ubiquitin gene: head-to-tail repeats encoding a polyubiquitin precursor protein. Nature 312 , 663-666.

Pao, K. C., Wood, N. T., Knebel, A., Rafie, K., Stanley, M., Mabbitt, P. D., et al. (2018). Activity-based E3 ligase profiling uncovers an E3 ligase with esterification activity. Nature 556, 381-385. doi: 10.1038/s41586-018-0026-1

Paraskevopoulos, K., Kriegenburg, F., Tatham, M. H., Rosner, H. I., Medina, B., Larsen, I. B., et al. (2014). Dss1 is a $26 \mathrm{~S}$ proteasome ubiquitin receptor. Mol. Cell 56, 453-461. doi: 10.1016/j.molcel.2014.09.008

Park, S. H., Bolender, N., Eisele, F., Kostova, Z., Takeuchi, J., Coffino, P., et al. (2007). The cytoplasmic Hsp70 chaperone machinery subjects misfolded and endoplasmic reticulum import-incompetent proteins to degradation via the ubiquitin-proteasome system. Mol. Biol. Cell 18, 153-165.

Peng, J., Schwartz, D., Elias, J. E., Thoreen, C. C., Cheng, D., Marsischky, G., et al. (2003). A proteomics approach to understanding protein ubiquitination. Nat. Biotechnol. 21, 921-926.

Pickart, C. M., Kasperek, E. M., Beal, R., and Kim, A. (1994). Substrate properties of site-specific mutant ubiquitin protein $(\mathrm{G} 76 \mathrm{~A})$ reveal unexpected mechanistic features of ubiquitin-activating enzyme (E1). J. Biol. Chem. 269, 7115-7123.

Pilli, M., Arko-Mensah, J., Ponpuak, M., Roberts, E., Master, S., Mandell, M. A., et al. (2012). TBK-1 promotes autophagy-mediated antimicrobial defense by controlling autophagosome maturation. Immunity 37, 223-234. doi: 10.1016/j. immuni.2012.04.015

Pinto, M. J., Pedro, J. R., Costa, R. O., and Almeida, R. D. (2016). Visualizing K48 ubiquitination during presynaptic formation by ubiquitination-induced fluorescence complementation (UiFC). Front. Mol. Neurosci. 9:43. doi: 10.3389/ fnmol.2016.00043

Pinto-Fernandez, A., Davis, S., Schofield, A. B., Scott, H. C., Zhang, P., Salah, E., et al. (2019). Comprehensive landscape of active deubiquitinating enzymes profiled by advanced chemoproteomics. Front. Chem. 7:592. doi: 10.3389/ fchem.2019.00592

Pruneda, J. N., Durkin, C. H., Geurink, P. P., Ovaa, H., Santhanam, B., Holden, D. W., et al. (2016). The molecular basis for ubiquitin and ubiquitin-like specificities in bacterial effector proteases. Mol. Cell 63, 261-276. doi: 10.1016/j. molcel.2016.06.015

Qiu, J., Sheedlo, M. J., Yu, K., Tan, Y., Nakayasu, E. S., Das, C., et al. (2016). Ubiquitination independent of E1 and E2 enzymes by bacterial effectors. Nature 533, 120-124. doi: 10.1038/nature17657

Rahighi, S., and Dikic, I. (2012). Selectivity of the ubiquitin-binding modules. FEBS Lett. 586, 2705-2710. doi: 10.1016/j.febslet.2012.04.053

Reyes-Turcu, F. E., Horton, J. R., Mullally, J. E., Heroux, A., Cheng, X., and Wilkinson, K. D. (2006). The ubiquitin binding domain ZnF UBP recognizes the C-terminal diglycine motif of unanchored ubiquitin. Cell 124, 1197-1208. 
Reyes-Turcu, F. E., Ventii, K. H., and Wilkinson, K. D. (2009). Regulation and cellular roles of ubiquitin-specific deubiquitinating enzymes. Annu. Rev. Biochem. 78, 363-397. doi: 10.1146/annurev.biochem.78.082307.091526

Reyes-Turcu, F. E., and Wilkinson, K. D. (2009). Polyubiquitin binding and disassembly by deubiquitinating enzymes. Chem. Rev. 109, 1495-1508.

Richter, B., Sliter, D. A., Herhaus, L., Stolz, A., Wang, C., Beli, P., et al. (2016). Phosphorylation of OPTN by TBK1 enhances its binding to Ub chains and promotes selective autophagy of damaged mitochondria. Proc. Natl. Acad. Sci. U.S.A. 113, 4039-4044. doi: 10.1073/pnas.1523926113

Roux, K. J., Kim, D. I., Raida, M., and Burke, B. (2012). A promiscuous biotin ligase fusion protein identifies proximal and interacting proteins in mammalian cells. J. Cell Biol. 196, 801-810. doi: 10.1083/jcb.201112098

Satpathy, S., Wagner, S. A., Beli, P., Gupta, R., Kristiansen, T. A., Malinova, D., et al. (2015). Systems-wide analysis of BCR signalosomes and downstream phosphorylation and ubiquitylation. Mol. Syst. Biol. 11:810. doi: 10.15252/msb. 20145880

Scott, D., Garner, T. P., Long, J., Strachan, J., Mistry, S. C., Bottrill, A. R., et al. (2016). Mass spectrometry insights into a tandem ubiquitin-binding domain hybrid engineered for the selective recognition of unanchored polyubiquitin. Proteomics 16, 1961-1969. doi: 10.1002/pmic.201600067

Shi, Y., Chan, D. W., Jung, S. Y., Malovannaya, A., Wang, Y., and Qin, J. (2011). A data set of human endogenous protein ubiquitination sites. Mol. Cell. Proteomics 10:M110002089. doi: 10.1074/mcp.M110.002089

Shiba-Fukushima, K., Imai, Y., Yoshida, S., Ishihama, Y., Kanao, T., Sato, S., et al. (2012). PINK1-mediated phosphorylation of the Parkin ubiquitin-like domain primes mitochondrial translocation of Parkin and regulates mitophagy. Sci. Rep. 2:1002. doi: $10.1038 /$ srep01002

Sims, J. J., Scavone, F., Cooper, E. M., Kane, L. A., Youle, R. J., Boeke, J. D., et al. (2012). Polyubiquitin-sensor proteins reveal localization and linkagetype dependence of cellular ubiquitin signaling. Nat. Methods 9, 303-309. doi: $10.1038 /$ nmeth. 1888

Sloper-Mould, K. E., Jemc, J. C., Pickart, C. M., and Hicke, L. (2001). Distinct functional surface regions on ubiquitin. J. Biol. Chem. 276, 30483-30489.

Stamenova, S. D., French, M. E., He, Y., Francis, S. A., Kramer, Z. B., and Hicke, L. (2007). Ubiquitin binds to and regulates a subset of SH3 domains. Mol. Cell 25, 273-284.

Swatek, K. N., and Komander, D. (2016). Ubiquitin modifications. Cell Res. 26, 399-422. doi: 10.1038/cr.2016.39

Swatek, K. N., Usher, J. L., Kueck, A. F., Gladkova, C., Mevissen, T. E. T., Pruneda, J. N., et al. (2019). Insights into ubiquitin chain architecture using Ub-clipping. Nature 572, 533-537. doi: 10.1038/s41586-019-1482-y

Tsuchiya, H., Burana, D., Ohtake, F., Arai, N., Kaiho, A., Komada, M., et al. (2018). Ub-ProT reveals global length and composition of protein ubiquitylation in cells. Nat. Commun. 9:524. doi: 10.1038/s41467-018-02869-x

Valkevich, E. M., Sanchez, N. A., Ge, Y., and Strieter, E. R. (2014). Middledown mass spectrometry enables characterization of branched ubiquitin chains. Biochemistry 53, 4979-4989. doi: 10.1021/bi5006305

van Wijk, S. J., Fiskin, E., Putyrski, M., Pampaloni, F., Hou, J., Wild, P., et al. (2012). Fluorescence-based sensors to monitor localization and functions of linear and K63-linked ubiquitin chains in cells. Mol. Cell 47, 797-809. doi: 10.1016/j.molcel.2012.06.017

van Wijk, S. J., Fulda, S., Dikic, I., and Heilemann, M. (2019). Visualizing ubiquitination in mammalian cells. EMBO Rep. 20:e46520. doi: 10.15252/embr. 201846520

Varshavsky, A. (2006). The early history of the ubiquitin field. Protein Sci. 15, 647-654.

Vasilescu, J., Smith, J. C., Ethier, M., and Figeys, D. (2005). Proteomic analysis of ubiquitinated proteins from human MCF-7 breast cancer cells by immunoaffinity purification and mass spectrometry. J. Proteome Res. 4, 2192-2200.

Vijay-Kumar, S., Bugg, C. E., and Cook, W. J. (1987). Structure of ubiquitin refined at 1.8 A resolution. J. Mol. Biol. 194, 531-544.

Wagner, S., Carpentier, I., Rogov, V., Kreike, M., Ikeda, F., Lohr, F., et al. (2008). Ubiquitin binding mediates the NF-kappaB inhibitory potential of ABIN proteins. Oncogene 27, 3739-3745. doi: 10.1038/sj.onc.1211042

Wagner, S. A., Beli, P., Weinert, B. T., Nielsen, M. L., Cox, J., Mann, M., et al. (2011). A proteome-wide, quantitative survey of in vivo ubiquitylation sites reveals widespread regulatory roles. Mol. Cell. Proteomics 10:M111013284. doi: 10.1074/mcp.M111.013284
Walden, H., and Rittinger, K. (2018). RBR ligase-mediated ubiquitin transfer: a tale with many twists and turns. Nat. Struct. Mol. Biol. 25, 440-445. doi: 10.1038/ s41594-018-0063-3

Walsh, C. T., Garneau-Tsodikova, S., and Gatto, G. J. Jr. (2005). Protein posttranslational modifications: the chemistry of proteome diversifications. Angew. Chem. 44, 7342-7372.

Walton, A., Stes, E., Cybulski, N., Van Bel, M., Inigo, S., Durand, A. N., et al. (2016). It's time for some "site"-seeing: novel tools to monitor the ubiquitin landscape in Arabidopsis thaliana. Plant Cell 28, 6-16. doi: 10.1105/tpc.15.00878

Wang, J., and Maldonado, M. A. (2006). The ubiquitin-proteasome system and its role in inflammatory and autoimmune diseases. Cell. Mol. Immunol. 3, 255-261.

Watt, G. F., Scott-Stevens, P., and Gaohua, L. (2019). Targeted protein degradation in vivo with proteolysis targeting chimeras: current status and future considerations. Drug Discov. Today Technol. 31, 69-80. doi: 10.1016/j.ddtec. 2019.02.005

Wauer, T., Simicek, M., Schubert, A., and Komander, D. (2015a). Mechanism of phospho-ubiquitin-induced PARKIN activation. Nature 524, 370-374.

Wauer, T., Swatek, K. N., Wagstaff, J. L., Gladkova, C., Pruneda, J. N., Michel, M. A., et al. (2015b). Ubiquitin Ser65 phosphorylation affects ubiquitin structure, chain assembly and hydrolysis. EMBO J. 34, 307-325. doi: 10.15252/embj. 201489847

Williams, C., van den Berg, M., Sprenger, R. R., and Distel, B. (2007). A conserved cysteine is essential for Pex4p-dependent ubiquitination of the peroxisomal import receptor Pex5p. J. Biol. Chem. 282, 22534-22543.

Winget, J. M., and Mayor, T. (2010). The diversity of ubiquitin recognition: hot spots and varied specificity. Mol. Cell 38, 627-635. doi: 10.1016/j.molcel.2010. 05.003

Witting, K. F., Mulder, M. P. C., and Ovaa, H. (2017). Advancing our understanding of ubiquitination using the Ub-toolkit. J. Mol. Biol. 429, 33883394. doi: 10.1016/j.jmb.2017.04.002

Xu, G., Paige, J. S., and Jaffrey, S. R. (2010). Global analysis of lysine ubiquitination by ubiquitin remnant immunoaffinity profiling. Nat. Biotechnol. 28, 868-873. doi: $10.1038 /$ nbt.1654

Xu, L., Fan, J., Wang, Y., Zhang, Z., Fu, Y., Li, Y. M., et al. (2019). An activity-based probe developed by a sequential dehydroalanine formation strategy targets HECT E3 ubiquitin ligases. Chem. Commun. 55, 7109-7112. doi: 10.1039/ c9cc03739j

$\mathrm{Xu}, \mathrm{P}$., and Peng, J. (2008). Characterization of polyubiquitin chain structure by middle-down mass spectrometry. Anal. Chem. 80, 3438-3444. doi: 10.1021/ ac800016w

Yang, C. S., Jividen, K., Spencer, A., Dworak, N., Ni, L., Oostdyk, L. T., et al. (2017). Ubiquitin modification by the E3 ligase/ADP-ribosyltransferase Dtx3L/Parp9. Mol. Cell 66, 503-516.e5. doi: 10.1016/j.molcel.2017.04.028

Yang, L., Chen, J., Huang, X., Zhang, E., He, J., and Cai, Z. (2018). Novel insights into E3 ubiquitin ligase in cancer chemoresistance. Am. J. Med. Sci. 355, 368-376. doi: 10.1016/j.amjms.2017.12.012

Yau, R. G., Doerner, K., Castellanos, E. R., Haakonsen, D. L., Werner, A., Wang, N., et al. (2017). Assembly and function of heterotypic ubiquitin chains in cellcycle and protein quality control. Cell 171, 918-933.e20. doi: 10.1016/j.cell.2017. 09.040

Zhang, X., Smits, A. H., van Tilburg, G. B., Jansen, P. W., Makowski, M. M., Ovaa, H., et al. (2017). An interaction landscape of ubiquitin signaling. Mol. Cell 65, 941-955.e8. doi: 10.1016/j.molcel.2017.01.004

Zhuang, M., Guan, S., Wang, H., Burlingame, A. L., and Wells, J. A. (2013). Substrates of IAP ubiquitin ligases identified with a designed orthogonal E3 ligase, the NEDDylator. Mol. Cell 49, 273-282. doi: 10.1016/j.molcel.2012.10. 022

Conflict of Interest: The authors declare that the research was conducted in the absence of any commercial or financial relationships that could be construed as a potential conflict of interest.

Copyright (c) 2020 Kliza and Husnjak. This is an open-access article distributed under the terms of the Creative Commons Attribution License (CC BY). The use, distribution or reproduction in other forums is permitted, provided the original author(s) and the copyright owner(s) are credited and that the original publication in this journal is cited, in accordance with accepted academic practice. No use, distribution or reproduction is permitted which does not comply with these terms. 